\title{
Optimal Surrender Strategies for Equity-Indexed Annuity Investors
}

\author{
Kristen S. Moore *
}

June 27, 2006

\begin{abstract}
An equity-indexed annuity (EIA) is a hybrid between a variable and a fixed annuity that allows the investor to participate in the stock market and earn at least a minimum interest rate. The investor sacrifices some of the upside potential for the downside protection of the minimum guarantee. Because EIAs allow investors to participate in equity growth without the downside risk, their popularity has grown rapidly.

An optimistic EIA owner might consider surrendering an EIA contract, paying a surrender charge, and investing the proceeds directly in the index to earn the full (versus reduced) index growth, while using a risk-free account for downside protection. Because of the popularity of these products, it is important for individuals and insurers to understand the optimal policyholder behavior.

We consider an EIA investor who seeks the surrender strategy and post-surrender asset allocation strategy that maximize the expected discounted utility of bequest. We formulate a variational inequality and a Hamilton-Jacobi-Bellman equation that govern the optimal surrender strategy and post-surrender asset allocation strategy, respectively. We examine the optimal strategies and how they are affected by the product features, model parameters, and mortality assumptions. We observe that in many cases, the "no-surrender" region is an interval $\left(w_{l}, w_{u}\right)$; i.e., that there are two free boundaries. In these cases, the investor surrenders the EIA contract if the fund value becomes too high or too low. In other cases, there is only one free boundary; the lower (or upper) surrender threshold vanishes. In these cases, the investor holds the EIA, regardless of how low (or high) the fund value goes. For a special case, we prove a succinct and intuitive condition on the model parameters that dictates whether one or two free boundaries exist.
\end{abstract}

Keywords: Optimal investment, optimal stopping, free boundary problem, equity-indexed annuity

JEL subject classification: C61, G11

*Department of Mathematics, University of Michigan, Ann Arbor, MI 48109-1109, fax: 7347630937. email:ksmoore@umich.edu 


\section{Introduction}

An equity-indexed annuity (EIA) is a hybrid between a fixed and variable annuity that allows the investor to participate in equity growth with limited downside risk. The investor pays a premium (or series of premiums) and the interest credited is linked to a benchmark index, typically the S\&P 500. The investor earns some fraction, called the participation rate, of the index growth and is guaranteed a minimum return on the premium. For example, a typical product offers the greater of $90 \%$ of the index growth or $3 \%$ per year on $90 \%$ of the premium invested. Effectively, the investor sacrifices some of the upside potential for the downside protection. Because of their "best of both worlds" design, EIAs are extremely popular. Sales have grown dramatically since their introduction in 1995. Indeed, EIA sales for 2005 were nearly $\$ 27.3$ billion, a $420 \%$ increase over their 2000 level of $\$ 5.25$ billion (The Advantage Compendium).

Because of their popularity, EIAs have received considerable attention in the actuarial literature; see, for example, Tiong (2000), Gerber and Pafumi (2000), Imai and Boyle (2001), Lee (2002), Gerber and Shiu (2003), Fung and Li (2003), and Lin and Tan (2003). These authors examine the pricing of various features of EIAs. We, on the other hand, are not pricing EIAs but rather are determining the optimal time for an individual to surrender a given EIA. Cheung and Yang (2005) study the optimal EIA surrender strategy in a discrete-time model with regime-switching.

A potential EIA investor might wonder whether she could outperform the EIA by investing directly in the index, thus earning the full, versus reduced growth, and a risk-free account for downside protection. Or, in a period of high returns, an EIA contract-holder might consider surrendering the contract, paying a surrender fee, and investing the proceeds on her own. Because of the popularity of these products, it is important for insurers and investors to understand the optimal policyholder behavior.

In this paper, we study the optimal surrender strategy for an EIA contract-holder. More specifically, we consider an investor who seeks to maximize her expected utility of bequest (or wealth at death) and study the optimal time to surrender the EIA contract, assuming that she allocates her assets optimally, in the sense of Merton (1992), after surrendering the contract. Moore and Young (2005) considered a similar problem for a perpetual EIA with time-homogeneous benefits under the assumption of constant hazard rate (i.e., exponential future lifetime). In that paper, we limited our study of the optimal strategies to the case in which lower and upper surrender thresholds exist.

In this paper, we extend that work by including time-dependent contract features, such as the minimum annual growth described above or the common time-dependent surrender fees included in EIAs. Moreover, while the constant hazard rate case yields valuable insight on the qualitative properties of the optimal strategies, this assumption is not a realistic model for human survival because of the memoryless property of the exponential distribution. We incorporate a more realistic, time-dependent hazard rate in our model and examine the impact of the mortality assumption on the optimal strategies. Our work complements the recent work of Cheung and Yang (2005) because, in order to focus on the effect of regime-swtiching, they ignore the effect of mortality and product features, such as 
the embedded guarantees. We focus on these assumptions and features.

Most significantly, we eliminate the a priori assumption of lower and upper surrender thresholds. We find that this structure exists in some cases; i.e., if the fund value is too low or too high, the investor should surrender the EIA and invest on her own. In other cases, there is no lower or upper threshold; the investor should keep the contract, regardless of the index performance. We examine the optimal strategy for a variety of product features and mortality assumptions. In the special case of of constant hazard rate and time-homogeneous benefits, we give a succinct condition for the existence of a lower threshold. For more general mortality and contract features, we compute the thresholds(s) numerically and observe similar phenomena.

\section{The Model}

Consider $(x)$, an investor aged $x$, whose utility of wealth $w$ is described by the increasing, concave, smooth utility function $u$. Let $T_{x}$ denote the future lifetime random variable for $(x)$ and let $\lambda_{x}$ denote the hazard rate function for $T_{x}$ (or the force of mortality for $(x)$ ).

Suppose the investor pays premium $\tilde{w}_{0}$ at time 0 to invest in an EIA and earns participation rate $\bar{p}$ of the index growth on the net deposit

$$
w_{0}:=\left(1-f_{0}\right) \tilde{w}_{0}
$$

less an annual maintenance fee at rate $f_{a}$ per year. The initial and maintenance fees $f_{0}$ and $f_{a}$ are called spread, asset, or margin fees and are similar to mutual fund fees.

We assume that the growth in the EIA investor's fund is linked to the performance of a stock or index whose price at time $t$ evolves according to the geometric Brownian motion

$$
d S_{t}=\mu S_{t} d t+\sigma S_{t} d B_{t}
$$

The process $B$ is a standard Brownian motion on a probability space $(\Omega, \mathcal{F}, \mathcal{P})$, and the constants $\mu$ and $\sigma$ are given positive constants. Note that we assume that the risky asset pays no dividends, or equivalently, that its price is constructed with all dividends reinvested. Thus, the evolution of the EIA fund value is governed by

$$
\left\{\begin{aligned}
d W_{t} & =\left(\bar{p} \mu-f_{a}\right) W_{t} d t+\bar{p} \sigma W_{t} d B_{t} \\
W_{0} & =w_{0}
\end{aligned}\right.
$$

At the surrender time $\tau_{s}$, the investor receives

$$
B\left(W_{\tau_{s}}, \tau_{s}\right):=\max \left\{\bar{s}(1+g)^{\tau_{s}} w_{0},\left(1-f_{s}\left(\tau_{s}\right)\right) W_{\tau_{s}}\right\}
$$

Here $g$ is the guaranteed minimum annual growth rate on a portion $\bar{s}$ of the initial (net) principal invested. A typical EIA product might offer a minimum of $3 \%$ per year on $90 \%$ of the principal invested; in this case, $g=0.03$ and $\bar{s}=0.90$. The time-dependent surrender fee is given by $f_{s}$; EIA products tend to have surrender fees that decrease to zero over a 7 to 17 year period. 
If the random time of death $\tau_{d}$ precedes the surrender time $\tau_{s}$, the contract pays the death benefit

$$
D\left(W_{\tau_{d}}, \tau_{d}\right):=\max \left\{\bar{d}(1+\tilde{g})^{\tau_{d}} w_{0}, W_{\tau_{d}}\right\} .
$$

If $g=\tilde{g}$, then the death benefit is simply the maximum of the fund value or the guaranteed minimum, with the surrender charge waived. On the other hand, if $\tilde{g}=0$ and $\bar{d}=1$, then the contract pays the maximum of the fund value or the initial (net) premium invested. Alternatively, if $\tilde{g}=0$, we could choose $\bar{d}$ so that $\bar{d} w_{0}$ is the amount of whole-life insurance that the initial net premium $w_{0}$ provides.

If the investor surrenders the EIA contract prior to death, i.e., if $\tau_{s}<\tau_{d}$, we assume that she invests in and trades dynamically between a risky and a risk-free account. We assume that the risky index is governed by the geometric Brownian motion (1); thus, she invests in the same index to which the EIA is linked. Moreover, we assume that the risk-free account grows at constant rate $r \in(0, \mu)$.

Let $\pi_{t}$ denote the dollar amount invested in the risky asset at time $t$. Then the evolution of the investor's wealth after surrendering the EIA is given by

$$
d W_{t}=\left(r W_{t}+(\mu-r) \pi_{t}\right) d t+\sigma \pi_{t} d B_{t} .
$$

The initial value of wealth is given by the EIA payout in (3). Thus, the investor's wealth is evolution is discontinuous; it is governed by (2) prior to the surrender time $\tau_{s}$ and by (5) after time $\tau_{s}$. The investor seeks the surrender time $\tau_{s}$ and the post-surrender asset allocations strategy $\pi_{t}$ to maximize the utility of wealth at the random time of death $\tau_{d}$. Jeanblanc, Lakner, and Kadam (2004) considered a similar optimal stopping problem with a discontinuous wealth evolution.

\subsection{Definition of the Value Functions}

In this section, we define the value functions for the pre- and post-surrender stochastic optimal control problems; we consider the post-surrender problem first. Given that the individual surrenders the EIA at time $\tau_{s}=t<\tau_{d}$ with wealth $W_{t}=w$, she is faced with a Merton problem of investment over a random horizon (Merton, 1992). Denote the value function of the Merton investment problem by $V$. Specifically, $V$ is given by

$$
V(w, t)=\sup _{\left\{\pi_{s}\right\} \in \mathcal{A}} E\left[e^{-\rho\left(\tau_{d}-t\right)} u\left(W_{\tau_{d}}\right) \mid W_{t}=w\right] .
$$

The set $\mathcal{A}$ is the set of admissible policies $\left\{\pi_{s}\right\}$ that are $\mathcal{F}_{s}$-progressively measurable, in which $\mathcal{F}_{s}$ is the augmentation of $\sigma\left(W_{u}: t \leq u \leq s\right)$, and that satisfy the integrability condition $E \int_{t}^{\tau_{d}} \pi_{s}^{2} d s<\infty$. We assume that the utility function $u: \mathbf{R} \rightarrow \mathbf{R}$ is increasing, concave, and smooth. The parameter $\rho$ is the individual's subjective discount rate; a large value of $\rho$ corresponds to a more impatient individual.

Assumption 2.1 Throughout this paper, we assume that preferences display constant relative risk aversion (CRRA); that is, $-w u^{\prime \prime}(w) / u^{\prime}(w)$ is a constant (Pratt, 1964). It follows 
that, up to an affine transformation, $u$ is given by a power function $u(w)=w^{1-\gamma} /(1-\gamma)$, in which $\gamma>0, \gamma \neq 1$, is the constant relative risk aversion. In most of our discussion, unless otherwise stated, we assume that $\gamma>1$; we comment on this choice below.

Several studies justify our choice of the relative risk aversion $\gamma>1$. See, for example, the well-cited article by Friend and Blume (1975), as well as the more recent Mitchell et al. (1999), Feldstein and Ranguelova (2001), Cochrane (2001), and Campbell and Viceira (2002).

Using the Dynamic Programming Principle and Itô's lemma, one can show that the value function $V$ solves the Hamilton-Jacobi-Bellman equation

$$
V_{t}+r w V_{w}+\max _{\pi}\left[(\mu-r) \pi V_{w}+\frac{1}{2} \sigma^{2} \pi^{2} V_{w w}\right]+\lambda_{x}(t) u(w)=V\left(\rho+\lambda_{x}(t)\right) .
$$

We refer the reader to Section 2.1 of Moore and Young (2005) for a discussion of the smoothness of solutions to (7). Standard verification theorems ensure that if (7) has a smooth solution $V$, then it equals the value function as defined in (6) and one can recover the the optimal investment strategy $\pi_{t}^{*}$ from the first order condition in (7). Moreover, the concavity of $u$ and the linearity of the dynamics (5) dictate that $V$ is concave in $w$. Thus, the maximum in (7) is well-defined and attained at

$$
\pi^{*}(w)=-\frac{\mu-r}{\sigma^{2}} \frac{V_{w}}{V_{w w}}
$$

Thus, we can rewrite (7) as

$$
V_{t}+r w V_{w}-\frac{1}{2} \frac{(\mu-r)^{2}}{\sigma^{2}} \frac{V_{w}^{2}}{V_{w w}}+\lambda_{x}(t) u(w)=V\left(\rho+\lambda_{x}(t)\right)
$$

Prior to surrendering the EIA, at any time $t<\tau_{d}$, the investor must decide whether to surrender the EIA or continue holding it. If the investor surrenders the EIA, she receives the payout $B\left(W_{\tau_{s}}, \tau_{s}\right)$ given in (3). Afterward, she invests optimally, as described above, and derives utility $V$. If the investor dies prior to surrender, she receives the death benefit $D\left(W_{\tau_{d}}, \tau_{d}\right)$ given in (4) and derives utility $u$. Thus, the value function prior to surrender is given by

$$
U(w, t)=\sup _{\tau_{s}} E\left[e^{-\rho\left(\tau_{s}-t\right)} V\left(B\left(W_{\tau_{s}}, \tau_{s}\right), \tau_{s}\right) 1_{\tau_{s}<\tau_{d}}+e^{-\rho\left(\tau_{d}-t\right)} u\left(D\left(W_{\tau_{d}}, \tau_{d}\right)\right) 1_{\tau_{d} \leq \tau_{s}} \mid W_{t}=w\right],
$$

where $1_{A}$ is the indicator function for the event $A$.

Again, using the Dynamic Programming Principle and Itô's lemma, one can show that $U$ solves the variational inequality

$$
\begin{gathered}
U_{t}+\left(\bar{p} \mu-f_{a}\right) w U_{w}+\frac{1}{2} \bar{p}^{2} \sigma^{2} w^{2} U_{w w}+\lambda_{x}(t) u(D(w, t)) \leq U\left(\rho+\lambda_{x}(t)\right), \\
U(w, t) \geq V(B(w, t), t)
\end{gathered}
$$


with equality holding in at least one of the two inequalities. We omit the details of the derivation, but refer the reader to Krylov (1980) or Oksendal (2000) for the background and to Young and Zariphopoulou (2002), Moore and Young (2003), and Young (2003) for similar derivations.

The intuition behind (11) and (12) is similar to the intuition for American options. If $U(w, t)>V(B(w, t), t)$, the expected utility from keeping the EIA exceeds the expected utility from surrendering; thus, the investor should not surrender the contract. The "surrender boundary" (or free boundary) is defined by the values of $(w, t)$ where the inequality in (12) switches to equality. In the no-surrender region, equality holds in (11) and inequality holds in (12).

Our goal in the work that follows is to find the optimal surrender time $\tau_{s}$ and the optimal post-surrender asset allocation strategy $\pi_{t}$. To find $\tau_{s}$, we seek the surrender boundary; i.e., we seek the values of $(w, t)$ where the inequality in (12) switches to equality. This prescribes the optimal surrender strategy; when the EIA fund value hits the free boundary, the investor should surrender the EIA contract and invest on her own according to the asset allocation strategy $\pi_{t}$.

Thus, we must solve the variational inequality (11), (12) and the HJB equation (9). In Sections 3 through 5, we consider these problems for three different cases:

- constant hazard rate and time-homogeneous benefits (Section 3)

- constant hazard rate and time-dependent benefits (Section 4)

- general hazard rate and time-dependent benefits (Section 5).

In the special case of Section 3, we establish conditions on the model parameters that govern the existence or non-existence of a lower surrender threshold. We prove this theorem in the Appendix in Section 8.

Throughout the examples, we assume that the EIA product is perpetual in the sense that the investor can continue to hold the contract (with the guarantees) indefinitely. There is precedent for considering perpetual products; see, for example, Gerber and Shiu (2003) and the references therein, as well as the comprehensive text on equity-indexed insurance by Hardy (2003). While this allows us to examine the qualitative properties of the free boundaries over long time, it is not a realistic assumption; most products have a fixed maturity date. Our model can be adapted easily to this case. We consider examples of fixed maturity products in Section 6. We find that the surrender strategy for the fixed maturity product is essentially a truncated version of the strategy for the perpetual product; the most significant differences in the strategies are intuitive and occur near the maturity time.

\section{Constant Hazard Rate, Time-Homogeneous Bene- fits}

In this section, we assume that the hazard rate is constant; i.e., that $\lambda_{x}(t)=\lambda$ for all $t$. Thus, we assume that the future lifetime $T_{x}$ of $(x)$ has exponential distribution with pa- 
rameter $\lambda$. Obviously, because of the memoryless property of the exponential distribution, this is not a valid model for human survival; however, this case yields valuable insight on the characterization of the surrender boundary.

Moreover, we assume that the contract features are independent of time; thus, in (3), we set $g=0$ and, without loss of generality, we set $f_{s}\left(\tau_{s}\right)=0$. (Note that we could easily set $f_{s}\left(\tau_{s}\right)$ to be any constant in $[0,1]$.) Similarly, in $(4)$, we set $\tilde{g}=0$.

This case was considered in Moore and Young (2005); however, in that paper, we restricted our analysis to the case in which the no-surrender region is an interval $\left(w_{l}, w_{u}\right)$; i.e., the case in which there are lower and upper surrender thresholds $w_{l}$ and $w_{u}$. In this section, we discuss cases for which there is no lower surrender threshold $w_{l}$. Moreover, we propose a succinct condition on the model parameters that determines whether one or two surrender thresholds exist. Friedman and Shen (2002) give a similar characterization for the optimal exercise boundary of an early retirement option.

Note that, under our assumptions above, the contract payout at the time of surrender is

$$
B\left(W_{\tau_{s}}, \tau_{s}\right)=B\left(W_{\tau_{s}}\right)=\max \left\{\bar{s} w_{0}, W_{\tau_{s}}\right\}
$$

and the death benefit is

$$
D\left(W_{\tau_{d}}, \tau_{d}\right)=D\left(W_{\tau_{d}}\right)=\max \left\{\bar{d} w_{0}, W_{\tau_{d}}\right\}
$$

Moreover, the value functions $V$ and $U$ are independent of time and the partial differential equations (PDEs) in (9) and (11) become ordinary differential equations (ODEs). Thus, $V$ solves the ODE

$$
r w V^{\prime}-\frac{1}{2} \frac{(\mu-r)^{2}}{\sigma^{2}} \frac{V^{\prime 2}}{V^{\prime \prime}}+\lambda u(w)=V(\rho+\lambda)
$$

and, in the no-surrender region, $U$ solves the free boundary problem for the ODE

$$
\left\{\begin{array}{c}
\left(\bar{p} \mu-f_{a}\right) w U^{\prime}+\frac{1}{2} \bar{p}^{2} \sigma^{2} w^{2} U^{\prime \prime}+\lambda u(D(w))=U(\rho+\lambda) \\
U(w) \geq V(B(w)) .
\end{array}\right\}
$$

It is straightforward to verify that the solution to (15) is given by

$$
V(w)=A \frac{w^{1-\gamma}}{1-\gamma}
$$

where

$$
A=\frac{\lambda}{\rho+\lambda-(r+m / \gamma)(1-\gamma)}
$$

and

$$
m=\frac{1}{2}\left(\frac{\mu-r}{\sigma}\right)^{2}
$$

Thus, by (8), the optimal asset allocation $\pi_{t}^{*}$ is given by

$$
\pi_{t}^{*}=\frac{1}{\gamma} \frac{\mu-r}{\sigma^{2}} W_{t}^{*}
$$


Note that this strategy is linear in the optimally controlled wealth $W_{t}^{*}$, as we expect for CRRA preferences. Note also that as the relative risk aversion $\gamma$ or index volatility $\sigma$ increase, the allocation to the risky asset decreases. As the excess return $\mu-r$ increases, the allocation to the risky asset increases. This is consistent with our financial intuition.

The solution to the ODE in (16) is given by

$$
U(w)= \begin{cases}C_{1} w^{a_{1}}+C_{2} w^{a_{2}}+\frac{\lambda}{\rho^{+\lambda}} \frac{\left(\bar{d} w_{0}\right)^{1-\gamma}}{1-\gamma}, & w \in\left(0, \bar{d} w_{0}\right) \\ \tilde{C}_{1} w^{a_{1}}+\tilde{C}_{2} w^{a_{2}}+\tilde{A}^{\frac{w^{1-\gamma}}{1-\gamma}}, & w \geq \bar{d} w_{0},\end{cases}
$$

where $a_{1}$ and $a_{2}$ are the positive and negative roots, respectively, of

$$
\frac{1}{2} \bar{p}^{2} \sigma^{2} a^{2}+\left(\bar{p} \mu-f_{a}-\frac{1}{2} \bar{p}^{2} \sigma^{2}\right) a-(\rho+\lambda)=0
$$

and $\tilde{A}$ is given by

$$
\tilde{A}=\frac{\lambda}{\rho+\lambda-\left(\bar{p} \mu-f_{a}\right)(1-\gamma)+\bar{p}^{2} \sigma^{2} \gamma(1-\gamma) / 2}
$$

In Moore and Young (2005), we postulated the existence of lower and upper surrender thresholds $w_{l}$ and $w_{u}$. In that paper, we computed the six unknowns $w_{l}, w_{u}, C_{i}, \tilde{C}_{i},(i=1,2)$ by assuming:

- that $U$ is continuous and smooth at $w=\bar{d} w_{0}$

- value-matching and smooth-pasting conditions at $w_{l}$ and $w_{u}$; i.e., that the value function $U$ meets the constraint function $V \circ B$ smoothly at $w_{l}$ and $w_{u}$.

These conditions gave six equations for the six unknowns. In that paper, we gave an algorithm for solving the system. The key step in the algorithm was finding a root $x=\frac{w_{u}}{w_{0}}$ of a function $\eta$ that may assume real or complex values; see equations (2.31) and (2.32) in Moore and Young (2005).

In this section, we consider cases for which the method of Moore and Young (2005) fails; i.e., cases for which $\eta$ has no real root (and hence the system of six equations in six unknowns has no solution).

In these cases, the function $U$ given in (19) above solves the free boundary problem (16). However, $C_{2}=0$ and there is no lower surrender threshold $w_{l}$. In these cases, the investor should continue to hold the EIA, regardless of how low the fund value drops.

Note that if $C_{2}=0$, we have from (19) that

$$
U(0)=\frac{\lambda}{\rho+\lambda} u(D(0))=\frac{\lambda}{\rho+\lambda} \frac{\left(\bar{d} w_{0}\right)^{1-\gamma}}{1-\gamma} .
$$

This result is consistent with our expectation; if one sets $w=0$ and "solves" the ODE in (16) for $U(0)$, this is the result. However, considering the definition of the value function 
$U$ in (10) yields a more convincing argument. If the fund value is zero, it stays at zero. Under constant hazard rate $\lambda$, we have that

$$
E\left[e^{-\rho \tau_{d}} u(D(0))\right]=u(D(0)) E\left[e^{-\rho \tau_{d}}\right]=\frac{\lambda}{\rho+\lambda} u(D(0)),
$$

as predicted.

Below, we propose a condition that determines whether a lower surrender threshold exists and we illustrate our result with numerical examples.

Definition 3.1 Let $Q=A \bar{s}^{1-\gamma}-\frac{\lambda}{\rho+\lambda} \bar{d}^{1-\gamma}$.

Remark 3.2 Assumption 8.2 specifies technical conditions on the model parameters. These assumptions are innocuous in the sense that they held for every "realistic" choice of parameters that we tested. We suggest that the reader ignore these technical conditions on the first reading.

Theorem 3.3 Assume that the relative risk aversion $\gamma>1$.

- If conditions (A1) and (A2) of Assumption 8.2 are satisfied and if $Q>0$, the free boundary problem (16) has only one free boundary $w_{u}$; the no-surrender region is the interval $\left(0, w_{u}\right)$.

- If conditions (A1)-(A4) of Assumption 8.2 are satisfied and if $Q<0$, the free boundary problem (16) has two free boundaries $0<w_{l}<w_{u}$; the no-surrender region is the interval $\left(w_{l}, w_{u}\right)$.

- If condition (A5) of Assumption 8.2 is satisfied and if $Q=0$, the lower surrender threshold is $w_{l}=0$.

Remark 3.4 If we assume that the relative risk aversion $\gamma \in(0,1)$, then the result above is reversed. When $Q>0$, there are two free boundaries and when $Q<0$ there is only one free boundary.

The idea behind the proof of the theorem is quite simple, but the proof is somewhat lengthy and heavy on notation; thus, we relegate it to the Appendix. The interpretation of the result is intuitively appealing. Assume that $\gamma>1$. Note that $Q>0$ when the death benefit proportion $\bar{d}$ is large relative to the minimum surrender guarantee proportion $\bar{s}$. Thus, because the death benefit is valuable, it is not surprising that the investor should not surrender the contract at low fund values. Moreover, by examining the sign of $\partial Q / \partial m$, we see that $Q>0$ when $m$ is small; i.e., when the excess return $\mu-r$ is small or the index volatility is large. Again, it is intuitive that an investor with a low fund value would not surrender the EIA contract and forego the protection of the minimum guarantee when the ratio of return to risk is too low. We comment on the interpretation of the result when $\gamma \in(0,1)$ in Experiment 3.3 below.

We remark that $Q$ is independent of the participation rate $\bar{p}$; thus, the existence of a lower surrender threshold does not depend on the participation rate. Moreover, $Q$ is independent of the initial and annual fees $f_{0}$ and $f_{a}$. (Note, however, that these parameters must be chosen to satisfy the conditions of Assumption 8.2.) 


\subsection{Numerical Examples}

We illustrate the result of Theorem 3.3 above with three numerical examples. We choose the following as our base scenario; it is similar to the base scenario studied in Moore and Young (2005).

- The relative risk aversion $\gamma=2$;

- The rate of return on the riskless asset $r=0.04$;

- The rate of return on the risky index $\mu=0.08$;

- The volatility on the risky asset $\sigma=0.2$;

- The participation rate $\bar{p}=0.9$;

- The proportion of the initial deposit returned upon death $\bar{d}=1.4$;

- The proportion of the initial deposit returned upon surrender $\bar{s}=0.9$;

- The force of mortality $\lambda=0.04$, so that the expected future lifetime of $(x+t)$ is 25 years for all $t>0$;

- The personal discount rate $\rho=0.04$;

- The initial premium invested is $\tilde{w}_{0}=1.0$;

- The initial fee $f_{0}=0.05$, so the initial net principal invested is $w_{0}=0.95$;

- The annual maintenance fee is $f_{a}=0$.

In each of the experiments that follow, we verify that $V\left(\tilde{w}_{0}\right)<U\left(w_{0}\right)$; i.e., that investing in the EIA increases the utility of the investor. Moreover, we verify that $w_{0} \in\left(w_{l}, w_{u}\right)$; i.e., that the investor is initially in the no-surrender region.

Experiment $3.1 Q<0$ versus $Q>0$ (Figure 1)

In this experiment, we contrast the results for the base scenario with the same scenario, but with $\lambda=0.055$. Our results are shown in Figure 1. The top graph shows the results for the base scenario. The dashed curve shows the constraint function $V \circ B$ and the solid curve shows the value function $U$. Since $Q=-0.0153<0$, we find upper and lower surrender thresholds $w_{l}=0.1387$ and $w_{u}=1.7721$. The no-surrender region is the interval $\left(w_{l}, w_{u}\right)$; if the fund value hits $w_{l}$ or $w_{u}$, the investor should surrender the EIA contract and invest on her own according the strategy $\pi_{t}^{*}$. Note that the value function and constraint functions are negative; this is because the utility $u$ is negative when $\gamma>1$; see Assumption 2.1. Note also that the value function meets the constraint function smoothly at $w_{l}$ and $w_{u}$ smoothly, as expected.

The bottom graph shows the results when we increase the hazard rate to $\lambda=0.055$. In this case, $Q=0.0079>0$; thus, there is only one surrender boundary $w_{u}=1.7859$. 

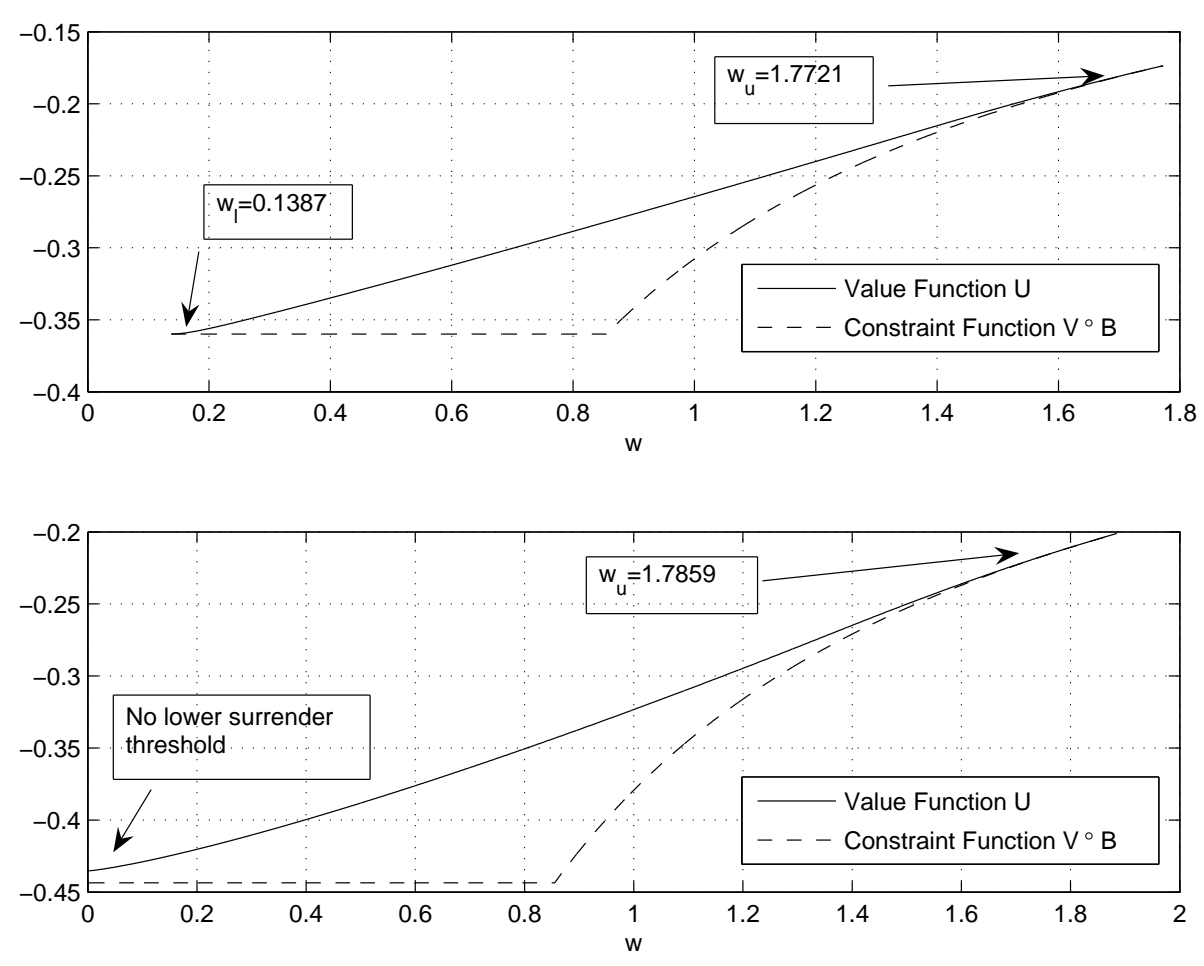

Figure 1: When $Q>0$, there is no lower surrender threshold.

In this case, the investor should hold the EIA contract regardless of how low the fund value drops, but surrender the contract when the fund value hits $w_{u}$. The loss of the lower surrender threshold is not surprising in this case. When the investor has higher hazard rate, and hence shorter expected future lifetime, the minimum guaranteed death benefit is more valuable at lower fund values than when the hazard rate is lower.

Experiment $3.2 Q<0$ versus $Q=0$ (Figure 2)

In this experiment, we consider the base scenario, but change the return on the riskless asset to $r=0.07$ and $r=0.02 \overline{6}$. In the first case, we have that $Q=-0.0621<0$ and the surrender thresholds are given by $w_{l}=0.4389$ and $w_{u}=1.3576$; see Figure 2 . In the second case, we have that $Q=0$ and the surrender thresholds are given by $w_{l}=0$ and $w_{u}=2.1728$. In both cases, the investor should hold the EIA contract while $w \in\left(w_{l}, w_{u}\right)$. It is intuitive that when $Q=0$, the lower surrender threshold occurs at $w_{l}=0 ; Q=0$ separates the case $Q>0$, for which no positive lower surrender threshold $w_{l}$ exists, from the case $Q<0$, for which positive $w_{l}$ does exist.

Experiment 3.3 Relative risk aversion $\gamma<1$ (Figure 3)

In Remark 3.4 above, we pointed out that if we choose $\gamma<1$, our result in Theorem 3.3 is reversed; when $Q>0$ there are two free boundaries and when $Q<0$ there is only one. We illustrate this with the following two examples. In the first, we choose the parameters in the base scenario, but we set $\gamma=0.8$. In this case, $Q=0.0498>0$. The top graph in Figure 3 shows that the surrender boundaries are $w_{l}=0.5506$ and $w_{u}=1.6248$. In the second example, we choose the same parameter values, but set $\lambda=0.15$. In this case, 

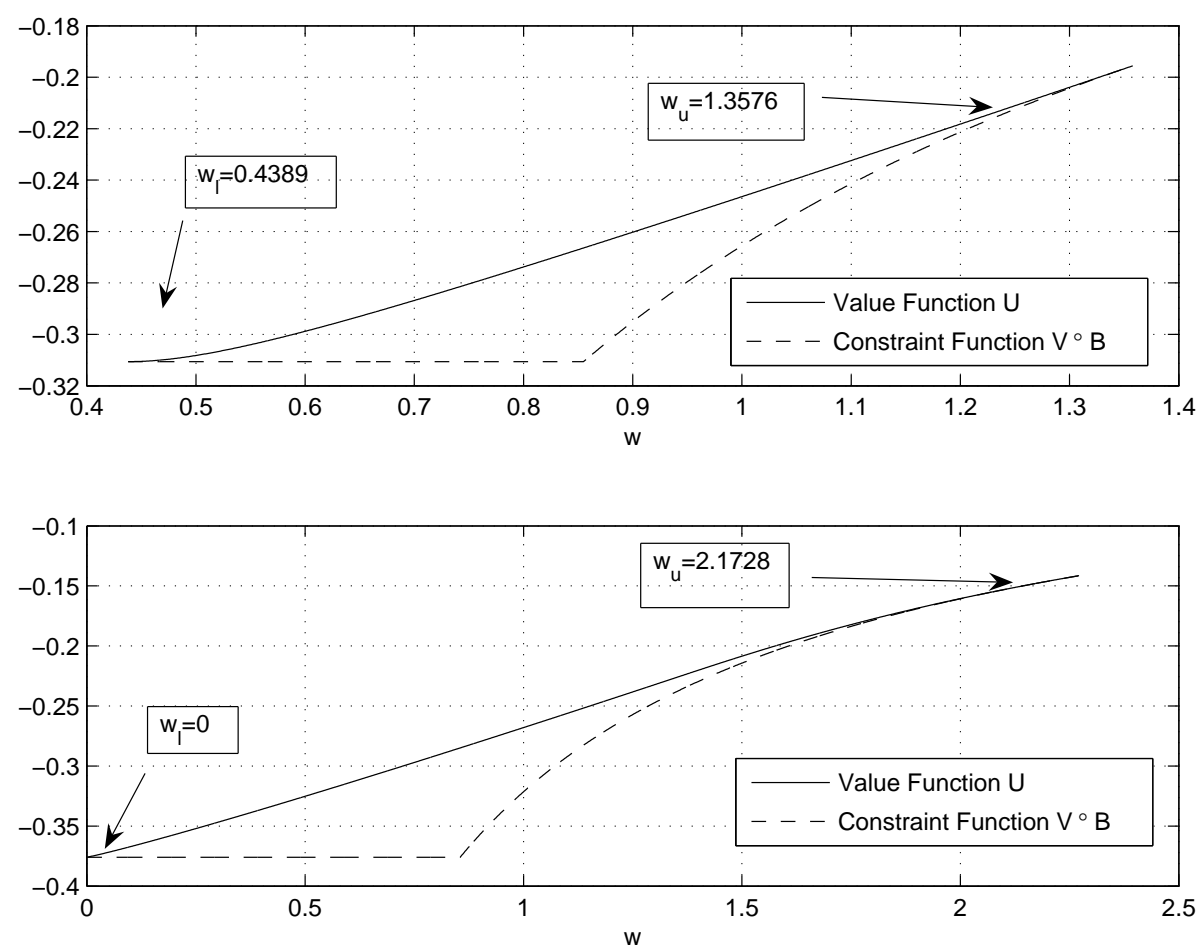

Figure 2: When $Q=0$, the lower surrender threshold occurs exactly at $w_{l}=0$.

$Q=-0.0146<0$. The bottom graph in Figure 3 shows that there is no lower surrender threshold and that the upper surrender threshold is $w_{u}=1.7797$.

The reversal of the result of Theorem 3.3 is not surprising. Indeed, note that when $\gamma<1$, negative values of $Q$ occur when, for example, $\bar{d}$ is large relative to $\bar{s}$. (Positive values of $Q$ occurred in this case when $\gamma>1$.) Since the death benefit is more valuable, the investor has incentive to continue to hold the EIA, even at low fund values. Similarly, when $\gamma \in(0,1)$, the sign of $\partial Q / \partial m$ confirms that negative values of $Q$ occur for small values of $m$; i.e., for small excess return $\mu-r$ or large index volatility $\sigma$. It is not surprising that an investor with low fund value would be unwilling to forego the minimum guarantee in such an environment.

\section{Constant Hazard Rate, Time-dependent Benefits}

In this section, we incorporate time-dependent benefits into the model and examine the impact of changing various product features on the surrender boundaries. In this case, the minimum guaranteed amount at surrender $B\left(W_{\tau_{s}}, \tau_{s}\right)$ and the minimum death benefit $D\left(W_{\tau_{d}}, \tau_{d}\right)$ depend on time and are given by equations (3) and (4). Note that since the force of mortality is constant, the post-surrender value function $V$ is still independent of time and is given by (17). However, the pre-surrender value function $U$ and the free boundaries depend on time. We must solve the variational inequality (11) and (12); more specifically, 

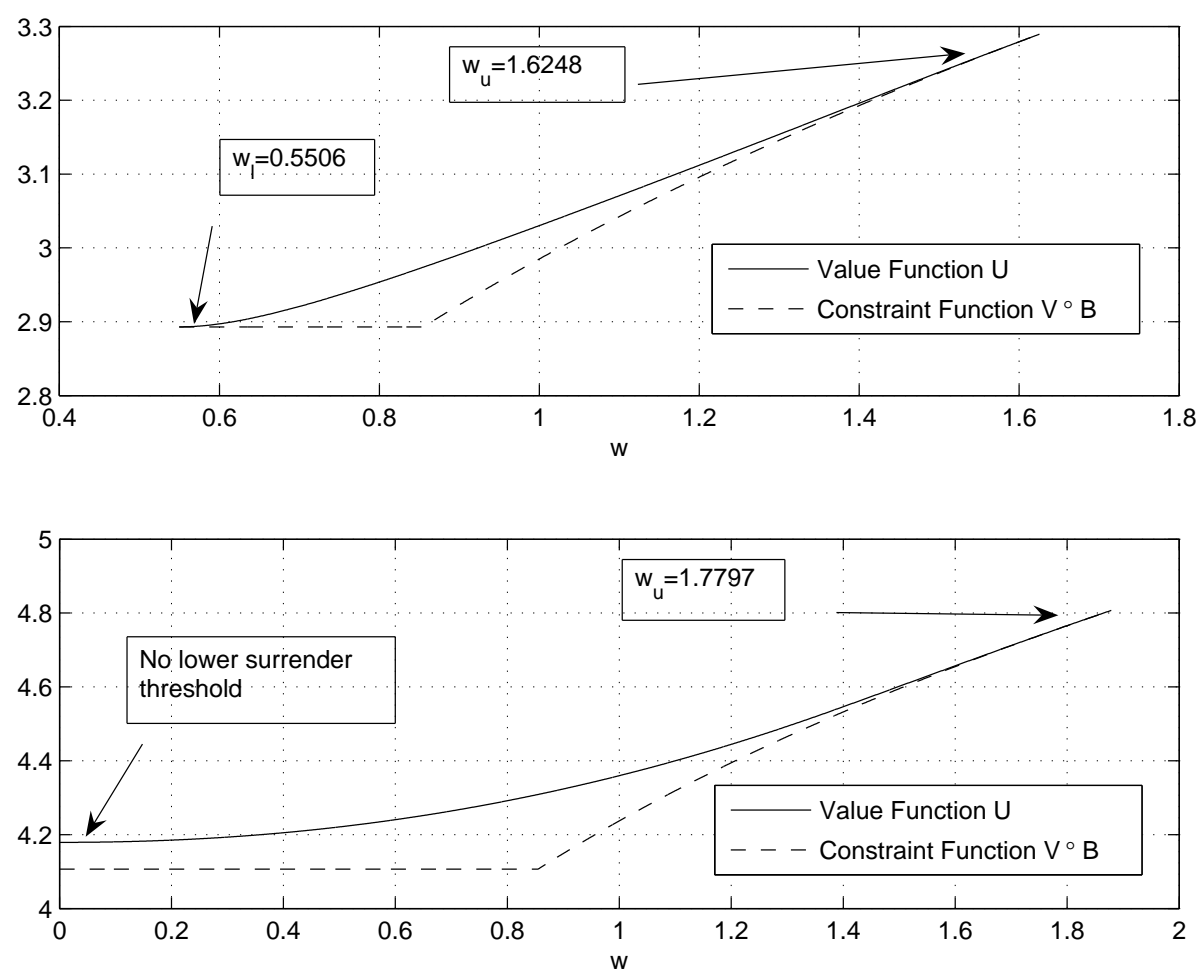

Figure 3: When $\gamma \in(0,1)$, there is no lower surrender threshold when $Q<0$.

in the no-surrender region, $U$ solves the free boundary problem for the PDE

$$
\left\{\begin{array}{c}
U_{t}+\left(\bar{p} \mu-f_{a}\right) w U_{w}+\frac{1}{2} \bar{p}^{2} \sigma^{2} w^{2} U_{w w}+\lambda u(D(w, t))=U(\rho+\lambda) \\
U(w, t) \geq V(B(w, t)) .
\end{array}\right\}
$$

(Recall that in Section 3, $U$ solved an ODE; in this case we must solve a PDE.)

Because this problem is similar to the free boundary problems that arise in American option pricing, we employ a similar numerical algorithm, namely, the Projected SOR Method. The Projected SOR Method is an iterative method for solving the PDE in (20) subject to the inequality constraint. We solve the constrained PDE on a domain that contains the free boundary and then recover the free boundary after computing the solution. The boundary points $w_{l}(t)$ and $w_{u}(t)$ are the points at which the inequality constraint in (20) switches to equality. We refer the reader to Wilmott, Dewynne, and Howison (2000) for more details.

We tested this method by using it to compute the value function and free boundaries for the ODE case of Section 3. The solutions computed via the projected SOR method matched the solution given by (19), so we are confident in the validity of our numerical scheme. 


\subsection{Numerical Experiments}

In the experiments that follow, we consider a base scenario and then examine the change in the surrender boundaries as we vary the product features. We choose the following as our base scenario; it is similar to the base scenario of Section 3.1.

- We choose the parameters $\gamma, r, \mu, \sigma, \bar{p}, \lambda, \rho$, and $\tilde{w}_{0}$ as in the base scenario of Section 3.1 .

- The initial fee $f_{0}=0$, so the initial net principal invested is $w_{0}=1$;

- The annual maintenance fee is $f_{a}=1.5 \%$;

- The minimum guaranteed growth rate (for death or surrender) is $3 \%$; i.e., $g=\tilde{g}=$ 0.03 ;

- The minimum guaranteed proportion of the initial deposit, accumulated at the minimum growth rate, returned upon death or surrender is $90 \%$; i.e., $\bar{s}=\bar{d}=0.9$;

- The surrender charge decreases linearly from $10 \%$ to $0 \%$ over a 10 -year period.

Thus, from the last three items above and equations (3) and (4), we have that the surrender and death benefits are given by

$$
\begin{aligned}
& B\left(W_{\tau_{s}}, \tau_{s}\right)=\max \left\{0.9(1.03)^{\tau_{s}} w_{0},\left(1-f_{s}\left(\tau_{s}\right)\right) W_{\tau_{s}}\right\} \\
& D\left(W_{\tau_{d}}, \tau_{d}\right)=\max \left\{0.9(1.03)^{\tau_{d}} w_{0}, W_{\tau_{d}}\right\}
\end{aligned}
$$

where

$$
f_{s}\left(\tau_{s}\right)=\left\{\begin{array}{cc}
0.01\left(10-\tau_{s}\right) & 0 \leq \tau_{s} \leq 10 \\
0 & \tau_{s}>10 .
\end{array}\right.
$$

Experiment 4.1 Results for the base scenario (Figures 4 and 5)

Figure 4 shows the value function $U$ (solid) and the constraint function $V \circ B$ (dashed) for $t=0,5,10$, and 15. The surrender boundaries $w_{l}$ and $w_{u}$ are the points at which $U=V \circ B$. For $w \in\left(w_{l}, w_{u}\right)$, the expected discounted utility from holding the EIA exceeds the expected discounted utility from surrendering the contract; thus, the investor should hold the EIA when the fund value is in $\left(w_{l}, w_{u}\right)$.

Figure 5 shows the evolution of the surrender boundaries for $t=0,1, \ldots, 30$. For example, we see that the left-hand surrender boundary (indicated by points) increases from $w_{l} \approx 0.657$ at $t=0$ to $w_{l} \approx 1.553$ at $t=30$. Note that the lower surrender threshold increases (i.e., the investor surrenders more readily at low fund value) as the minimum guaranteed amount (indicated by squares) increases. Note also that the upper surrender boundary (indicated by asterisks) decreases during the 10-year surrender charge period; thus, the investor surrenders less readily when the surrender charge is higher (at $t=0$, for example) and more readily when the surrender charge is lower (at $t=9$, for example). Beyond the surrender charge period, the upper surrender threshold increases with the minimum guaranteed amount. 

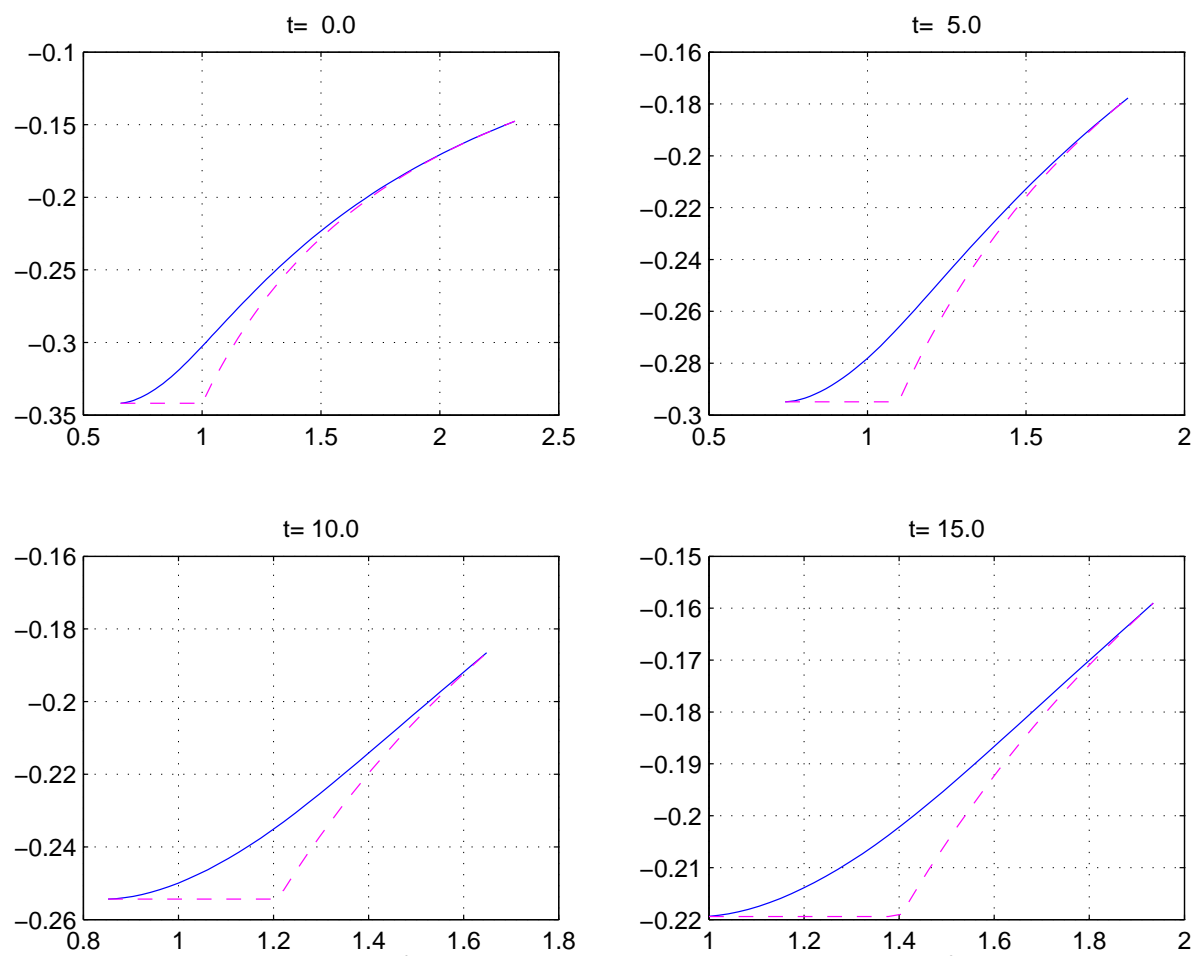

Figure 4: The value function $U$ and the constraint function $V \circ B$ for the base scenario.

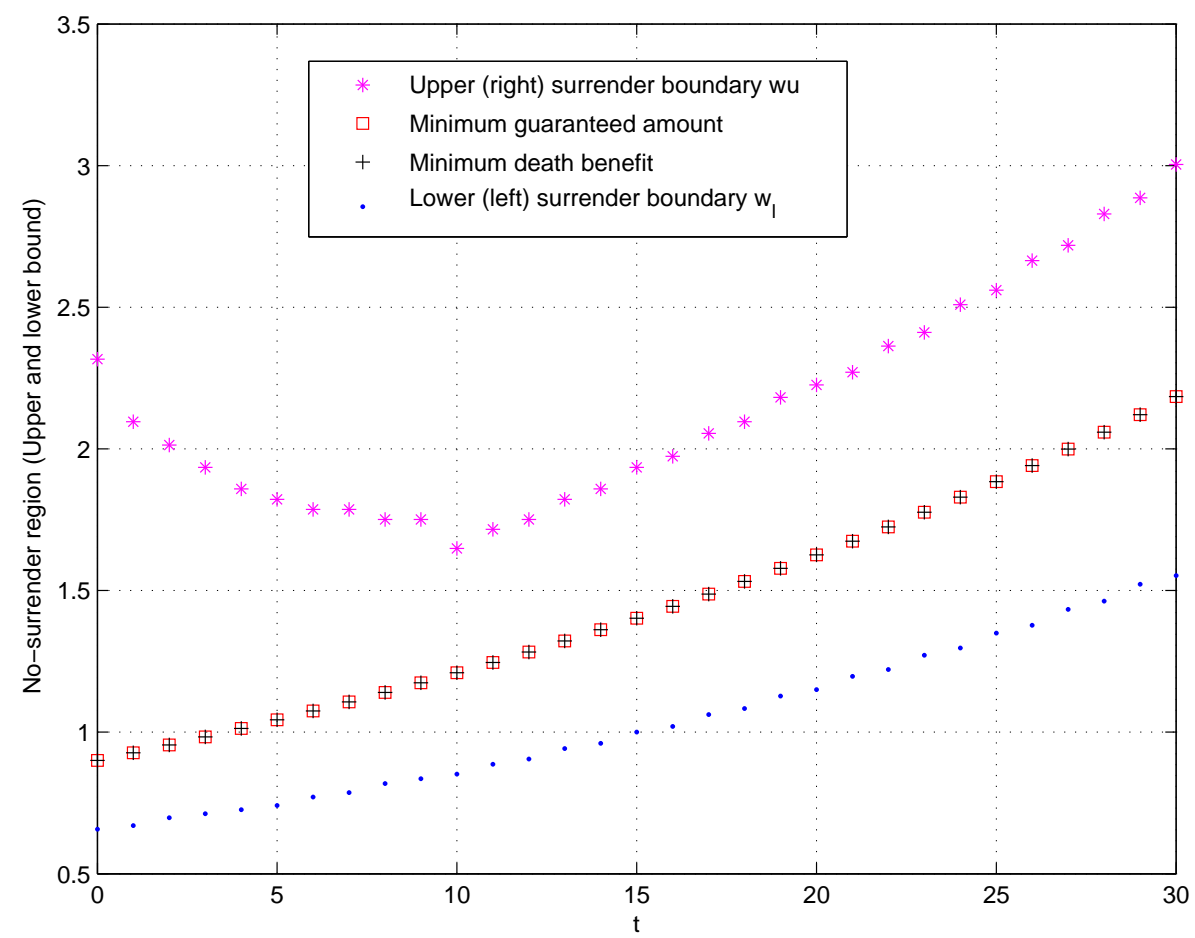

Figure 5: The evolution of the free boundary for the base scenario 

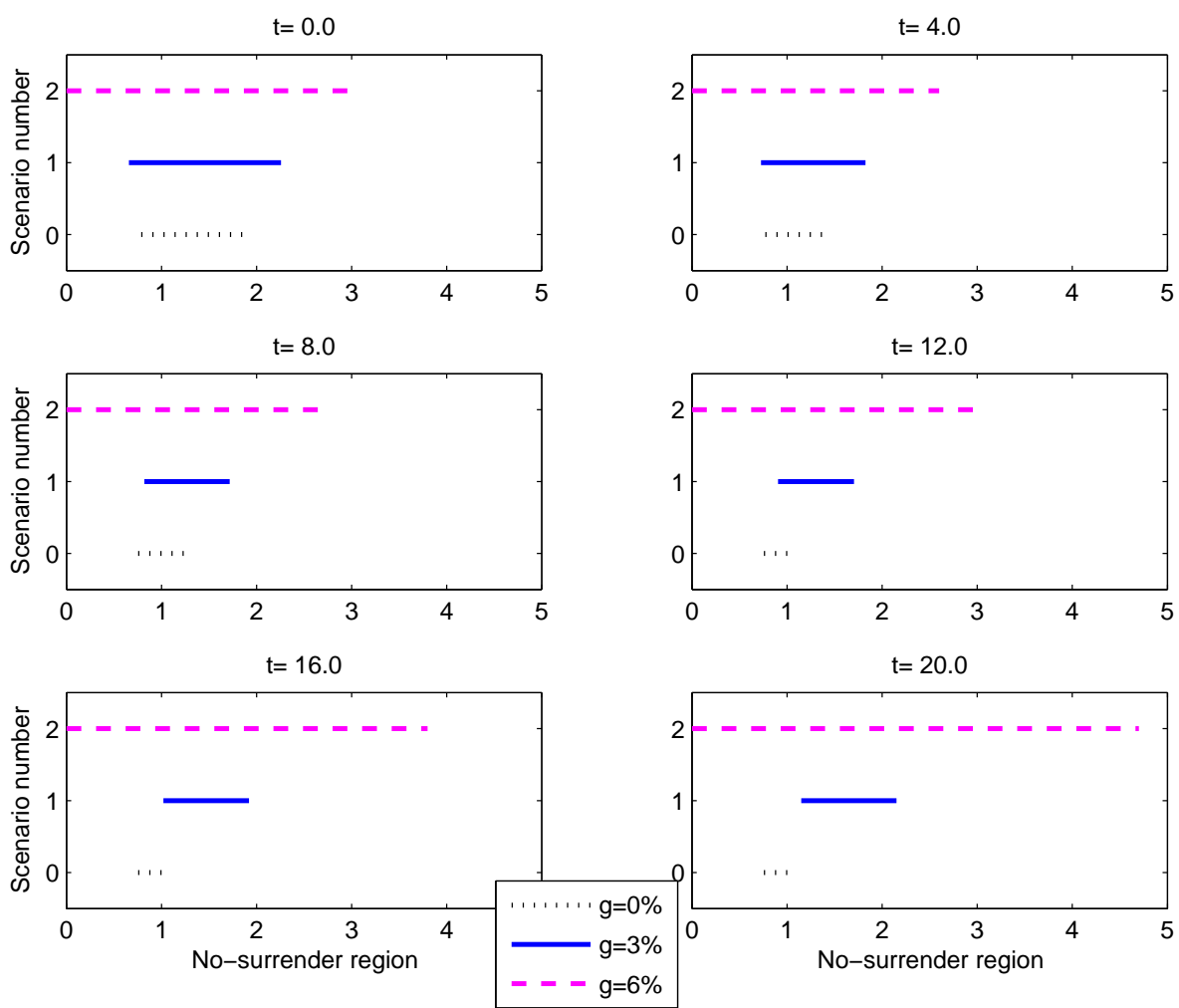

Figure 6: The investor surrenders more readily when the guaranteed minimum growth rate is lower.

Experiment 4.2 Impact of changing the guaranteed minimum growth rates (Figure 6) In this experiment, we examine the impact of changing the guaranteed minimum growth rates $g$ and $\tilde{g}$. Figure 6 contrasts the surrender boundaries for the following three scenarios.

- Scenario 0: $g=\tilde{g}=0 \%($ dotted line $)$

- Scenario 1: Base scenario: $g=\tilde{g}=3 \%$ (solid line)

- Scenario 2: $g=\tilde{g}=6 \%$ (dashed line)

The vertical axis indicates the number of the scenario while the horizontal axis shows the no-surrender region $\left(w_{l}, w_{u}\right)$. It is not surprising to see that the investor surrenders less readily (i.e., the no-surrender region is wider) when the minimum guaranteed growth rate is higher. In fact, when the minimum growth rate is $6 \%$, there is no lower surrender threshold $w_{l}$ for $t \in[0,20]$; the investor will not forego the protection of the minimum guarantee, regardless of how low the fund value drops. On the other hand, when $g=\tilde{g}=0$, the no-surrender region is much narrower; the investor surrenders more readily at low and high fund values.

Experiment 4.3 Impact of changing the maximum surrender charge (Figure 7) In this experiment, we consider three different surrender fee structures. 
- Scenario 0: no surrender charge (dotted line)

- Scenario 1: the surrender charge decreases linearly from $5 \%$ to $0 \%$ over 10 years (solid line)

- Scenario 2: Base Scenario: the surrender charge decreases linearly from $10 \%$ to $0 \%$ over 10 years (dashed line)

In Figure 7, first note that at $t=12,16$, and 20, beyond the 10-year surrender charge period, the surrender boundaries are the same for all three scenarios. This is intuitive as the surrender charge no longer applies for these values of $t$. During the surrender charge period $(t=0,4$, and 8$)$, we see that the no-surrender region is wider (i.e., the investor surrenders less readily) when the surrender charge is is higher. Note also that the left surrender boundaries are not affected by the change in surrender charge. This is because the left surrender boundary $w_{l}$ is smaller than the minimum guaranteed amount. When the investor surrenders at $w=w_{l}$, she receives the minimum guaranteed amount, but the surrender charge does not apply to this amount; see equation (3). However, the right-hand surrender boundary $w_{u}$ is affected by the change in surrender charge during the surrender charge period. This is because $w_{u}$ exceeds the minimum guaranteed amount; thus, when the investor surrenders at $w=w_{u}$, she receives the fund value $w$, reduced by the surrender charge. These results are consistent with our intuition.

Experiment 4.4 Impact of changing the surrender charge period (Figure 8)

In this experiment, we examine the impact of changing the surrender charge period. In each scenario, the initial surrender charge is $10 \%$ and it decreases linearly to $0 \%$ over 5 , 10, or 15 years. Thus, our three scenarios are:

- Scenario 0: 5-year surrender charge period (dotted line)

- Scenario 1: Base scenario: 10-year surrender charge period (solid line)

- Scenario 2: 15-year surrender charge period (dashed line)

In Figure 8, we see that the left-hand surrender boundaries are unaffected by the change in the surrender charge period at all times and that the right hand boundaries are unaffected beyond the surrender charge period; we explained these phenomena in the previous experiment. At times $t=8$ and $t=12$, we see another unsurprising phenomenon: the investor surrenders more readily if she is closer to the end of (or past) the surrender charge period, when the surrender charge is lower (or zero).

However, at time $t=0$, we see a surprising phenomenon. Though the surrender charge at time zero is $10 \%$ in all three scenarios, the right-hand surrender boundary differs markedly. In fact, it vanishes completely in scenario 0; when the surrender charge period is only 5 years, at time $t=0$, there is no upper surrender threshold $w_{u}$. Thus, in this case, the investor should continue to hold the EIA, regardless of how high the fund value grows. Because the surrender charge decreases more rapidly, the investor can avoid the higher surrender charge by waiting for a few years. We note that a finite right-hand surrender 

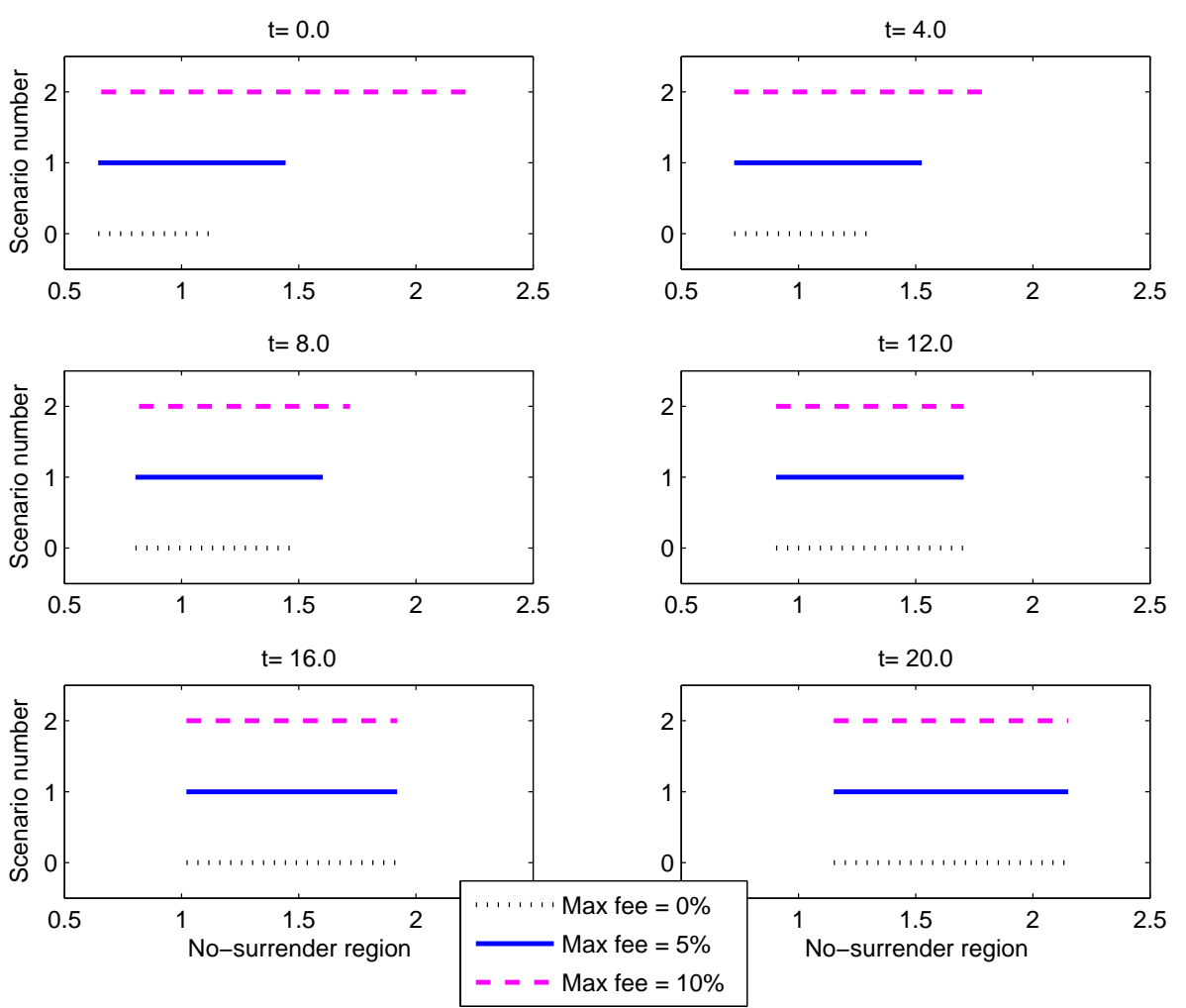

Figure 7: During the 10-year surrender charge period, the investor surrenders more readily when the surrender charge is lower. 

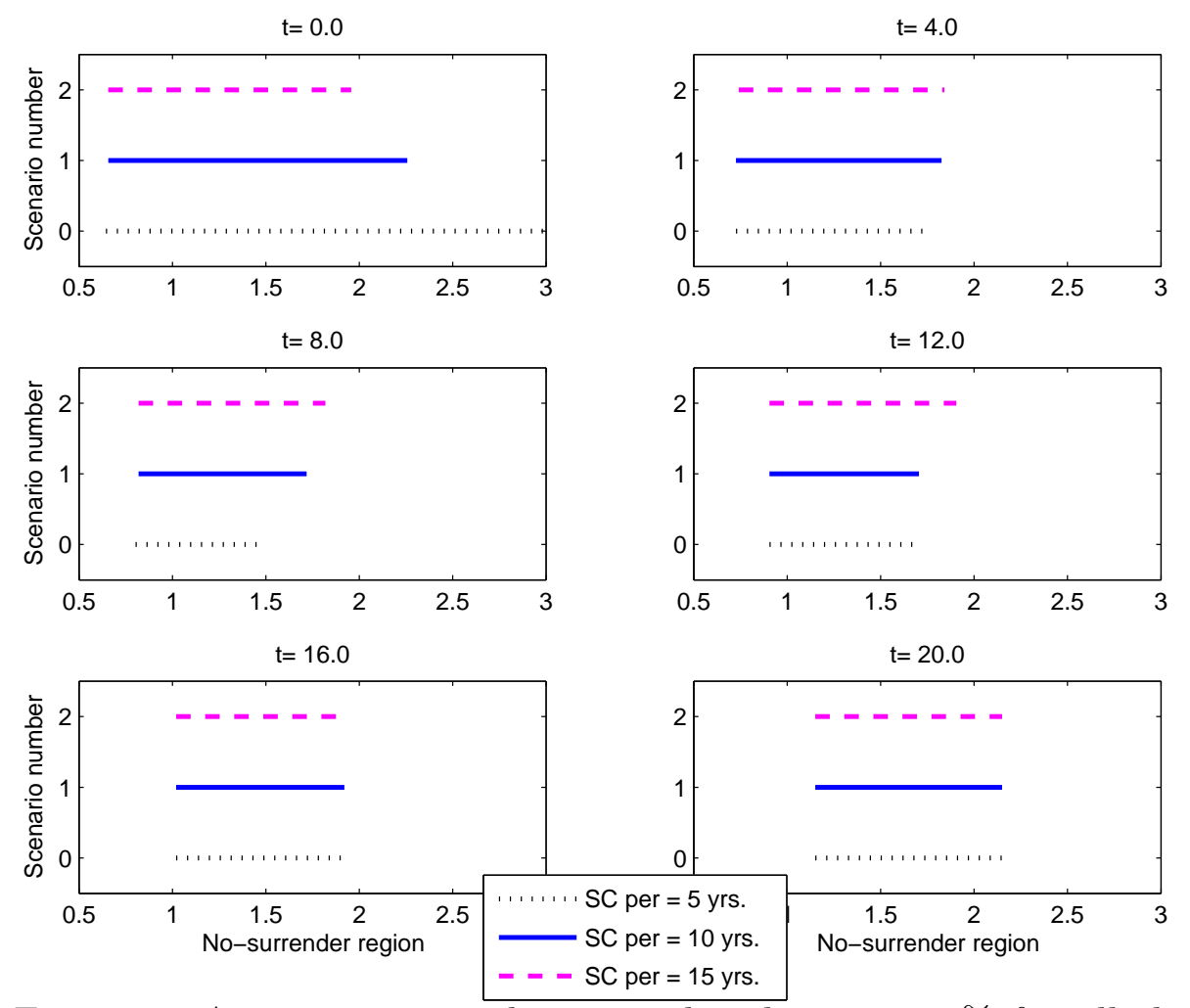

Figure 8: At time $t=0$, the surrender charge is $10 \%$ for all three scenarios; however, the right-hand free boundaries differ markedly. In particular, there is no right-hand free boundary for Scenario 0 at time $t=0$.

boundary appears by time $t=4$. (In fact, it happens some time in $(3,4)$.) We note that this is the first experiment in which the right-hand surrender boundary vanishes.

Experiment 4.5 Impact of changing the participation rate (Figure not shown) In this experiment, we examine the impact of changing the participation rate $\bar{p}$. We consider the following three scenarios:

- Scenario 0: $\bar{p}=80 \%$ (dotted line)

- Scenario 1: Base scenario: $\bar{p}=90 \%$ (solid line)

- Scenario 2: $\bar{p}=100 \%$ (dashed line)

We find that the no-surrender region is wider when the participation rate is higher; there is greater incentive to hold the contract when $\bar{p}$ is higher. Moreover, we see that the nosurrender region narrows (i.e., the investor surrenders more readily) as the surrender charge decreases. Finally, the no surrender region moves to the right as the guaranteed minimum amount increases. This is consistent with our earlier results.

Experiment 4.6 Impact of changing the annual fee (Figure 9)

In this experiment, we examine the impact of changing the annual fee $f_{a}$. We consider the following two scenarios. 

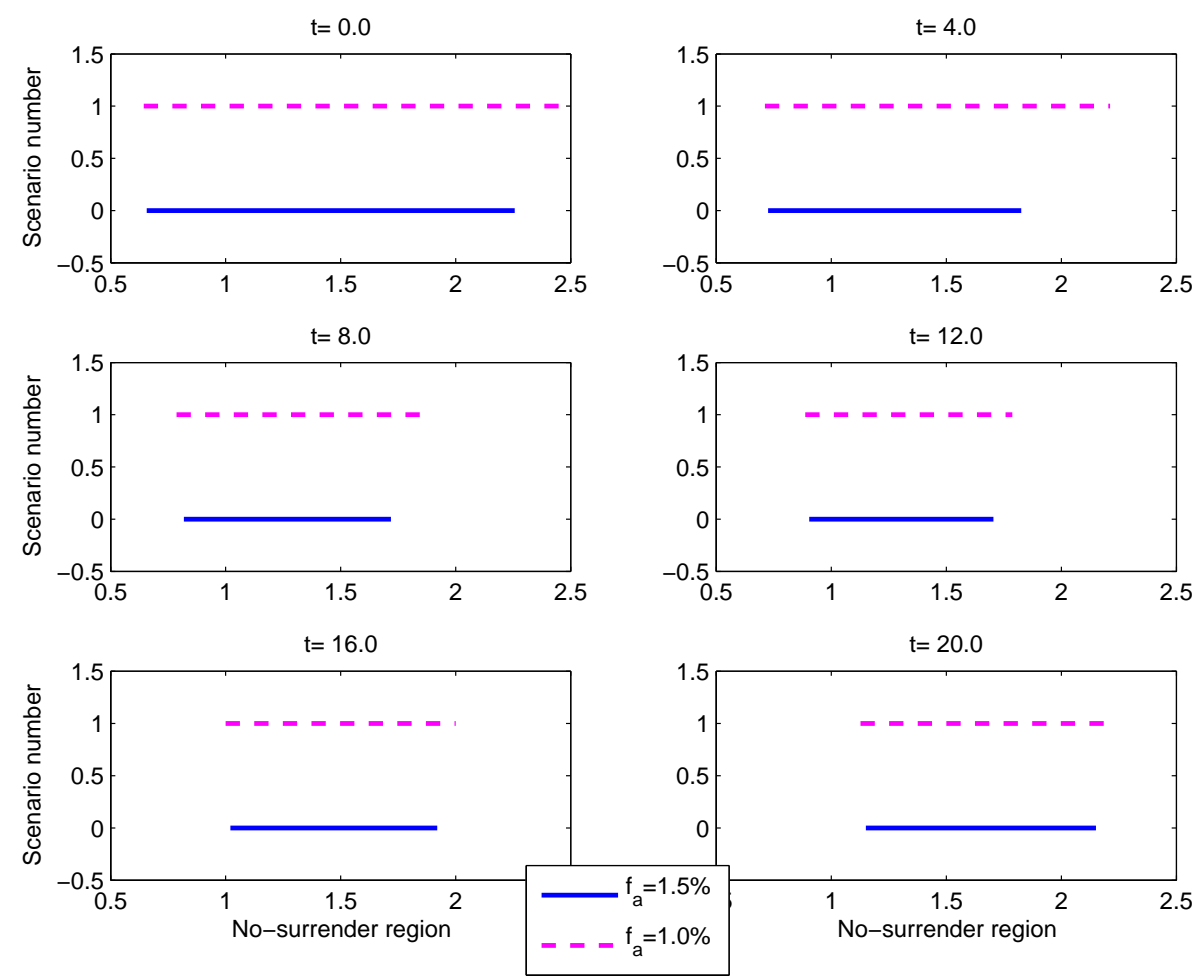

Figure 9: When the annual fee is lower, there is no upper surrender threshold at time $t=0$; the finite right-hand free boundary appears by time $t=4$, when the surrender charge is lower.

- Scenario 0: Base scenario: $f_{a}=1.5 \%$ (solid line)

- Scenario 1: $f_{a}=1.0 \%$ (dashed line)

Figure 9 shows that the investor should hold the contract longer when the annual fee is lower and that the difference in the surrender boundaries is more pronounced during the 10-year surrender charge period. Moreover, at time $t=0$, there is no upper surrender threshold $w_{u}$ when $f_{a}=1.0 \%$; the investor should continue to hold the EIA, regardless of how high the fund value goes. At the lower annual fee, the EIA is a "good deal;" thus, the investor should continue to hold the contract until the surrender charge decreases somewhat. Note that a finite right-hand surrender boundary appears by time $t=4$. (In fact, it happens some time in $(2,3)$.) This is our second experiment in which the right-hand free boundary vanishes. (See Experiment 4.4.)

Experiment 4.7 Impact of changing the death benefit (Figure 10) In this experiment, we examine the impact of changing the structure of the death benefit. We consider the following two scenarios.

- Scenario 0: Base scenario: $D\left(W_{\tau_{d}}, \tau_{d}\right)=\max \left\{0.9(1.03)^{\tau_{d}} w_{0}, W_{\tau_{d}}\right\}$ (solid line)

- Scenario 1: $D\left(W_{\tau_{d}}, \tau_{d}\right)=\max \left\{1.4 w_{0}, W_{\tau_{d}}\right\}$ (dashed line) 

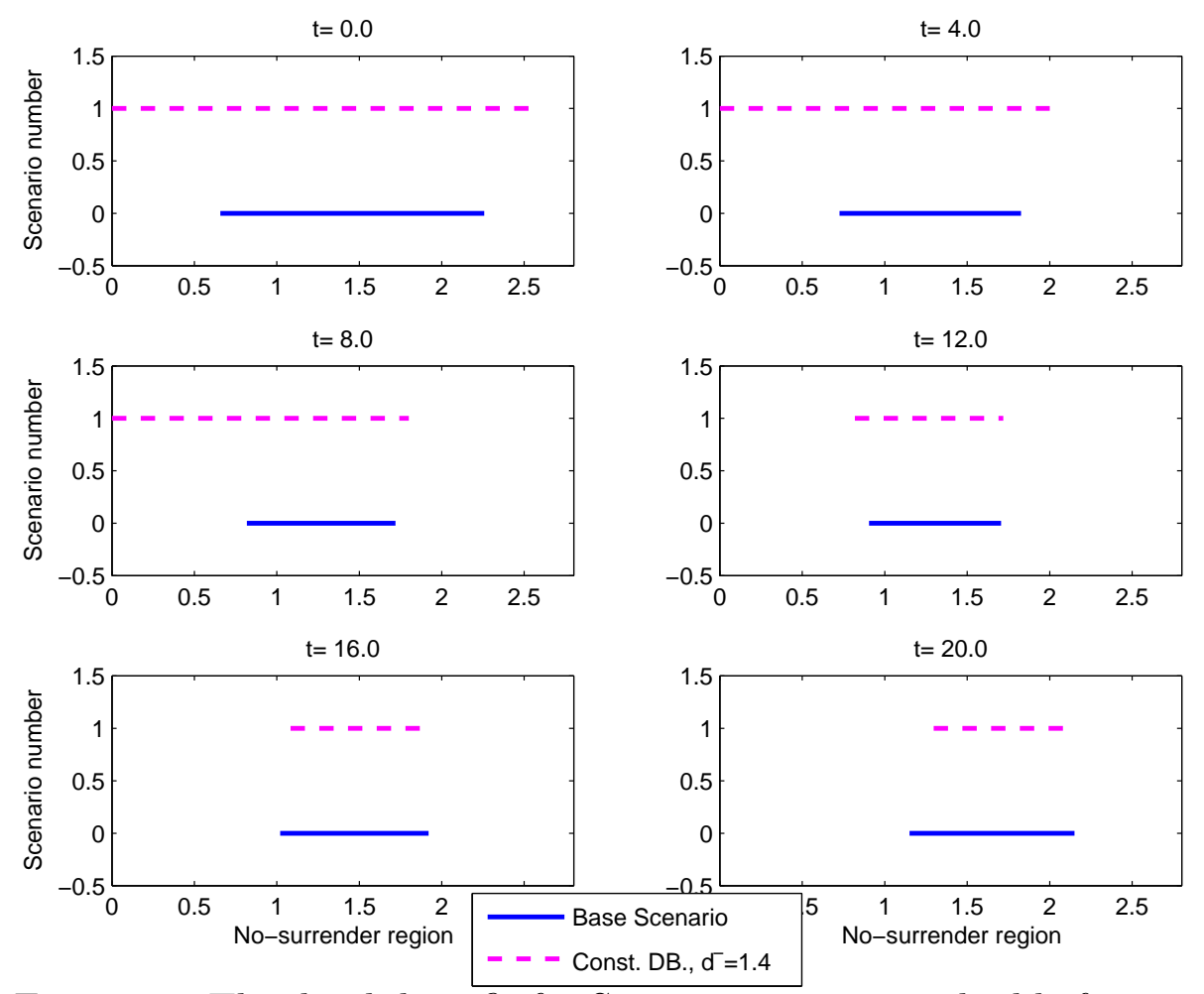

Figure 10: The death benefit for Scenario 1 is more valuable for $t<14.95$ and less valuable for $t>14.95$; the investor surrenders the product with the less valuable death benefit more readily.

Thus, we contrast the base scenario, in which the death benefit is simply the guaranteed minimum amount at surrender with a waiver of surrender charges, with a death benefit whose minimum guaranteed amount is a fixed proportion of the initial net deposit $w_{0}$. Note that the two minimum death benefits are equal if $\tau_{d} \approx 14.95$.

In Figure 10, we see that at times 0,4,8, and 12 the no-surrender region is wider (i.e., the investor surrenders less readily) in Scenario 1. This is because the guaranteed minimum death benefit for Scenario 1 is higher when $t<14.95$. For $t=16$ and $t=20$, the investor surrenders less readily in Scenario 0; this is because the time-dependent guaranteed minimum death benefit is more valuable for larger $t$. Note also that in Scenario 1, there is no left-hand surrender boundary for $t=0,4$, and 8; i.e., the investor should not surrender the contract, regardless of how low the fund value drops. A non-zero left-hand free boundary appears by time $t=10$. This is because the surrender charge is costly and the higher minimum guaranteed death benefit is more valuable at low fund values. These results are consistent with our intuition. 


\section{General Hazard Rate}

So far, we have considered only constant hazard rate $\lambda_{x}(t)=\lambda$ for all $t$. Under this assumption, the value function $V$, which governs the post-surrender asset allocation strategy, solves the ODE (15) and is given in closed form by (17). In this section, we study the investor's optimal strategies under more realistic forms of the hazard rate. In this case, we must solve the PDE (9) for $V$ and then use $V \circ B$ as the constraint function in solving the variational inequality (11), (12).

Again, it is straightforward to verify that the solution to (9) is given by

$$
V(w, t)=\xi(t) u(w)
$$

where $\xi$ solves the ODE

$$
\xi^{\prime}=\left[\rho+\lambda_{x}(t)-\left(r+\frac{m}{\gamma}\right)(1-\gamma)\right] \xi-\lambda_{x}(t) .
$$

Note that if $\lambda_{x}(t)=\lambda$ is constant, $\xi(t)=A$ solves the ODE (22), where $A$ is given by (18). Thus, we recover the closed form solution of Section 3 in the case of constant hazard rate.

We remark that $\xi$ can also be computed directly from the definition of the value function $V$ in (6). First note that since $V$ is given by (21) and $\pi^{*}$ is given by (8), we have that

$$
\pi_{t}^{*}=\frac{\mu-r}{\gamma \sigma^{2}} W_{t}^{*}
$$

where $W_{t}^{*}$ is the optimally controlled wealth. Thus, by (5), we have that

$$
\begin{aligned}
d W_{s}^{*} & =\left[r W_{s}^{*}+(\mu-r) \pi_{s}^{*}\right] d s+\sigma \pi_{s}^{*} d B_{s} \\
& =\left[r+\frac{(\mu-r)^{2}}{\gamma \sigma^{2}}\right] W_{s}^{*} d s+\frac{\mu-r}{\gamma \sigma} W_{s}^{*} d B_{s} .
\end{aligned}
$$

Suppose an investor has wealth $w$ at time $t$. Solving the stochastic differential equation above we have that for $s>t$

$$
\begin{aligned}
W_{s}^{*} & =w \exp \left\{\left(r+\frac{(\mu-r)^{2}}{\gamma \sigma^{2}}-\frac{1}{2} \frac{(\mu-r)^{2}}{\gamma^{2} \sigma^{2}}\right)(s-t)+\frac{\mu-r}{\gamma \sigma} B_{s-t}\right\} \\
& =w \exp \{\underbrace{\left(r+\frac{(\mu-r)^{2}}{\gamma \sigma^{2}} \frac{2 \gamma-1}{2 \gamma}\right)}_{\alpha_{1}}(s-t)+\underbrace{\frac{\mu-r}{\gamma \sigma}}_{\alpha_{2}} B_{s-t}\} \\
& =w \exp \left\{\alpha_{1}(s-t)+\alpha_{2} B_{s-t}\right\} .
\end{aligned}
$$

Now, from the definition (6) of $V$, we have that

$$
\begin{aligned}
V(w, t) & =E\left[e^{-\rho\left(\tau_{d}-t\right)} u\left(W_{\tau_{d}}^{*}\right) \mid W_{t}=w\right] \\
& =E\left[e^{-\rho\left(\tau_{d}-t\right)} \frac{\left[w \exp \left\{\alpha_{1}\left(\tau_{d}-t\right)+\alpha_{2} B_{\tau_{d}-t}\right\}\right]^{1-\gamma}}{1-\gamma}\right]
\end{aligned}
$$




$$
\begin{aligned}
& =\underbrace{\frac{w^{1-\gamma}}{1-\gamma}}_{u(w)} \underbrace{E\left[e^{-\rho\left(\tau_{d}-t\right)} e^{\alpha_{1}(1-\gamma)\left(\tau_{d}-t\right)} e^{\left.\alpha_{2}(1-\gamma) B_{\tau_{d}-t}\right]}\right.}_{\xi(t)} \\
& =u(w) \xi(t) ;
\end{aligned}
$$

i.e., that

$$
\xi(t)=E\left[e^{-\rho\left(\tau_{d}-t\right)} e^{\alpha_{1}(1-\gamma)\left(\tau_{d}-t\right)} e^{\alpha_{2}(1-\gamma) B_{\tau_{d}-t}}\right],
$$

where $\alpha_{1}$ and $\alpha_{2}$ are defined in the calculation (23).

Now, $\tau_{d}=\tau$ is the future lifetime random variable of $(x)$ given that she has survived until time $t$; denote the probability density function of $\tau$ by $f_{\tau}$. The conditional distribution of $Y=B_{\tau-t}$ given $\tau$ is normal with mean zero and variance $\tau-t$; denote the probability density function of this random variable by $\varphi(y)$. We can compute the expectation in (24) directly by integrating against the normal density times $f_{\tau}$. Letting $M_{Y}$ denote the moment generating function of the random variable $Y$, we have that

$$
\begin{aligned}
\xi(t) & =\int_{-\infty}^{\infty} \int_{t}^{\infty} \exp \left\{\left[-\rho+\alpha_{1}(1-\gamma)\right](\tau-t)\right\} \exp \left[\alpha_{2}(1-\gamma) y\right] f_{\tau}(\tau) \varphi(y) d \tau d y \\
& =\int_{t}^{\infty} \exp \left\{\left[-\rho+\alpha_{1}(1-\gamma)\right](\tau-t)\right\} f_{\tau}(\tau) \underbrace{\int_{-\infty}^{\infty} \exp \left[\alpha_{2}(1-\gamma) y\right] \varphi(y) d y}_{M_{Y}\left(\alpha_{2}(1-\gamma)\right)} d \tau \\
& =\int_{t}^{\infty} \exp \left\{\left[-\rho+\alpha_{1}(1-\gamma)+\frac{\alpha_{2}^{2}(1-\gamma)^{2}}{2}\right](\tau-t)\right\} f_{\tau}(\tau) d \tau \\
& =\int_{t}^{\infty} \exp \left\{\left[-\rho+(1-\gamma)\left(r+\frac{m}{\gamma}\right)\right](\tau-t)\right\} f_{\tau}(\tau) d \tau .
\end{aligned}
$$

Note that $\rho>(1-\gamma)(r+m / \gamma)$ implies that $\xi \leq 1$, and from (21) it follows that $V(w, t) \leq$ $u(w)$ for all $t$. That is, if the investor's personal discount rate is sufficiently high, her optimal expected future discounted utility from current wealth $w$ is smaller than her immediate utility from wealth $w$. Note also that if $\lambda_{x}(t)=\lambda$ is constant, so that $f_{\tau}(\tau)=\lambda e^{-\lambda(\tau-t)}$, we have that $\xi(t)=A$, as before.

It turns out that evaluating $\xi$ via the formulations (22) and (25) is numerically delicate for "realistic" $\lambda_{x}(t)$. In particular, we choose a Gompertz (exponential) hazard rate $\lambda_{x}(t)$; we explain this choice below in Section 5.1. The "initial" condition for the ODE (22) is specified at $T=\infty$. But since $\lambda_{x}(t)$ is large for large $t$, some numerical solvers fail. Moreover, the choice of large $T$ (i.e., the "numerical value" of $T=\infty$ ) affects the solutions; the placement of the far-field condition affects the near-field solution. In addition, under Gompertz hazard rate, the integrand in the last line of (25) approaches a delta function as $t \rightarrow \infty$, thus many standard numerical integration routines fail. We evaluate $\xi$ as follows:

- use the integral formulation in the last line of $(25)$ to evaluate $\xi(T)$ for some "large" $T$

- use this value of $\xi(T)$ as the terminal condition for the ODE (22) and solve the ODE for $\xi$ via a Runge-Kutta solver

- during the same time-stepping procedure, use the Projected SOR Method described in Section 4 to solve the free boundary problem for $U$. 

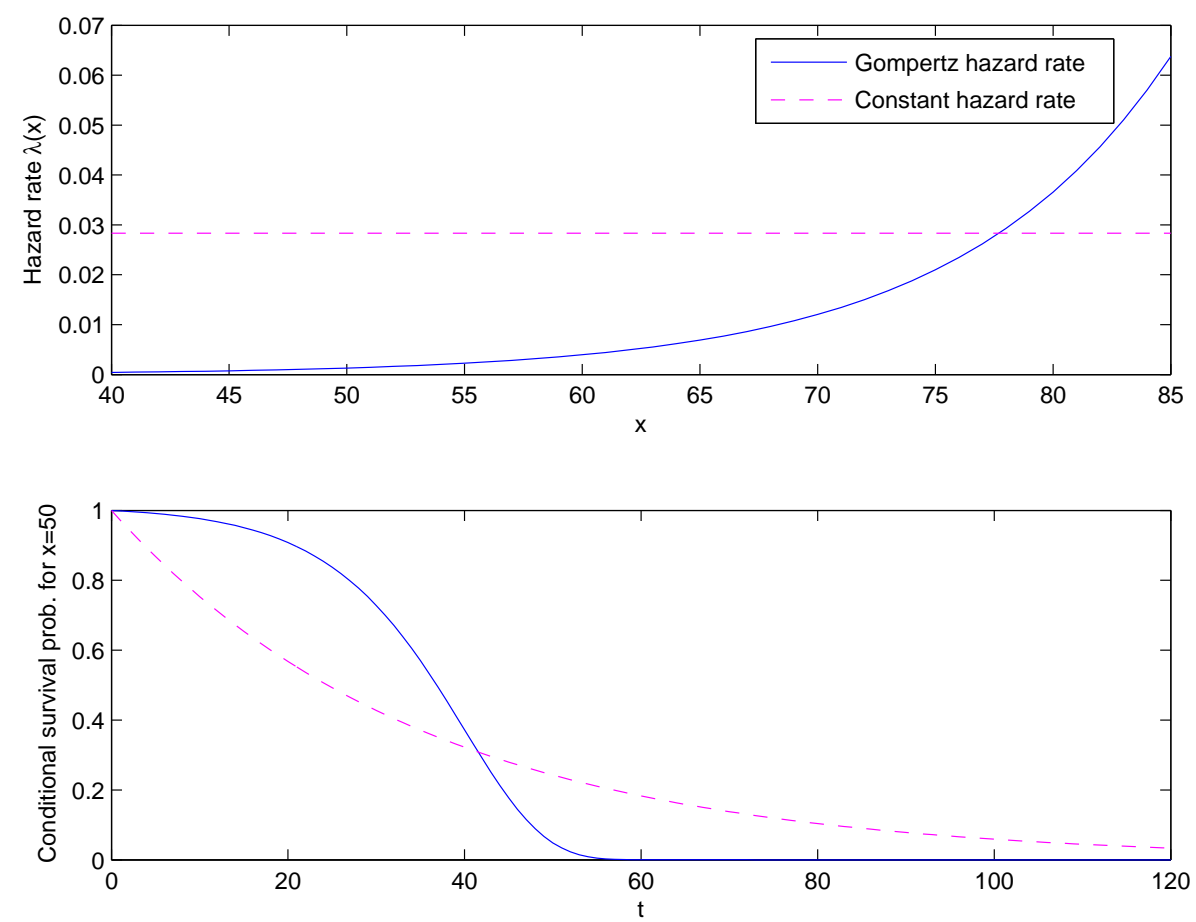

Figure 11: The two hazard rate assumptions yield the same expected future lifetime for a 50-year-old. The conditional survival probabilities differ significantly.

\subsection{Numerical Experiments}

In this section, we examine the impact of the more realistic mortality assumption. Consistent with the mortality assumptions in Milevsky and Young (2003) and Huang, Milevsky, and Wang (2004), we use the Gompertz hazard rate

$$
\lambda_{x}(t)=\frac{1}{b} \exp \left(\frac{x+t-\bar{m}}{b}\right) .
$$

We choose $\bar{m}=90$ and $b=9$ to approximate the Individual Annuity Mortality 2000 (basic) Table with projection scale $\mathrm{G}$.

We consider two experiments: one for which the product features are independent of time and one for which they are time-dependent. In both experiments, we contrast the Gompertz hazard rate described above with the constant hazard rate $\lambda=0.0283$. Under both assumptions, the expected future lifetime for a 50-year-old is 35.3 years. Figure 11 shows both mortality assumptions; the top graph shows the hazard rate and the bottom graph shows ${ }_{t} p_{50}$, the conditional probability that a 50-year-old survives another $t$ years, given that she has survived to age 50. Observe that under the Gompertz hazard rate, the conditional survival probabilities are higher for small $t$ and lower for large $t$ than under the constant hazard rate.

In both experiments, we choose the parameters $\gamma, r, \mu, \sigma, \bar{p}, \rho, \tilde{w}_{0}, f_{0}$, and $f_{a}$ as in the base scenario of Section 3.1. We describe the minimum guarantees and surrender charges in the experiments below. 
Experiment 5.1 Product features are independent of time (Figure 12) In this experiment, as in Section 3, we consider the case in which the product features do not depend on time so that we can isolate the impact of the time dependent hazard rate $\lambda_{x}(t)$. In particular, we choose the minimum guaranteed amount and death benefit as in the base scenario of Section 3.1, so that

$$
B\left(W_{\tau_{s}}, \tau_{s}\right)=B\left(W_{\tau_{s}}\right)=\max \left\{0.9 w_{0}, W_{\tau_{s}}\right\}
$$

and

$$
D\left(W_{\tau_{d}}, \tau_{d}\right)=D\left(W_{\tau_{d}}\right)=\max \left\{1.4 w_{0}, W_{\tau_{d}}\right\} .
$$

Figure 12 shows the minimum guaranteed amount and death benefit (indicated by squares and plus signs, respectively), the lower and upper free boundaries under the Gompertz hazard rate (indicated by triangles), and the lower and upper free boundaries under the constant hazard rate (indicated by points and asterisks, respectively) for $t=0,1, \ldots, 30$. We see that for $t \leq 24$, the no-surrender region is much narrower under the Gompertz hazard rate; i.e., the Gompertz investor surrenders the product more readily. However, for larger $t$, the Gompertz investor becomes more conservative and continues to hold the contract over a wider range of fund values. In particular, for $t \geq 29$, the lower free boundary vanishes; the Gompertz investor continues to hold the EIA, regardless of how low the fund value drops.

Even though both mortality assumptions yield the same future life expectancy $\stackrel{\circ}{e}_{50}$ at age 50, under the Gompertz hazard rate $\stackrel{\circ}{e}_{50+t}$ decreases with $t$. Under the constant hazard rate, $\stackrel{\circ}{e}_{50+t}=\stackrel{\circ}{e}_{50} \approx 35.3$ for all $t$. Moreover, as we observed in the bottom graph of Figure 11, for larger $t$, the conditional survival probabilities are lower for the Gompertz hazard rate than for the constant hazard rate. Thus, as $t$ increases, the death benefit becomes more valuable to the Gompertz investor and she surrenders less readily.

Experiment 5.2 Time-dependent product features (Figure 13)

In this experiment, we allow the product features to vary with time. We choose the minimum guaranteed amount, death benefit, and surrender charges as in the base scenario of Section 4.1 , so that

$$
\begin{aligned}
& B\left(W_{\tau_{s}}, \tau_{s}\right)=\max \left\{0.9(1.03)^{\tau_{s}} w_{0},\left(1-f_{s}\left(\tau_{s}\right)\right) W_{\tau_{s}}\right\} \\
& D\left(W_{\tau_{d}}, \tau_{d}\right)=\max \left\{0.9(1.03)^{\tau_{d}} w_{0}, W_{\tau_{d}}\right\}
\end{aligned}
$$

and

$$
f_{s}\left(\tau_{s}\right)=\left\{\begin{array}{cc}
0.01\left(10-\tau_{s}\right) & 0 \leq \tau_{s} \leq 10 \\
0 & \tau_{s}>10 .
\end{array}\right.
$$

Figure 13 shows the minimum guaranteed amount and death benefit as well as the lower and upper surrender boundaries under both mortality assumptions. We see that the change in the mortality assumption has little impact on the free boundaries for most values of $t$; though the Gompertz investor is slightly more conservative, the free boundaries are close. Moreover, we see that no upper surrender threshold exists under either assumption for $t=0,1, \ldots, 7$; the investor will not surrender the contract, regardless of how high the 


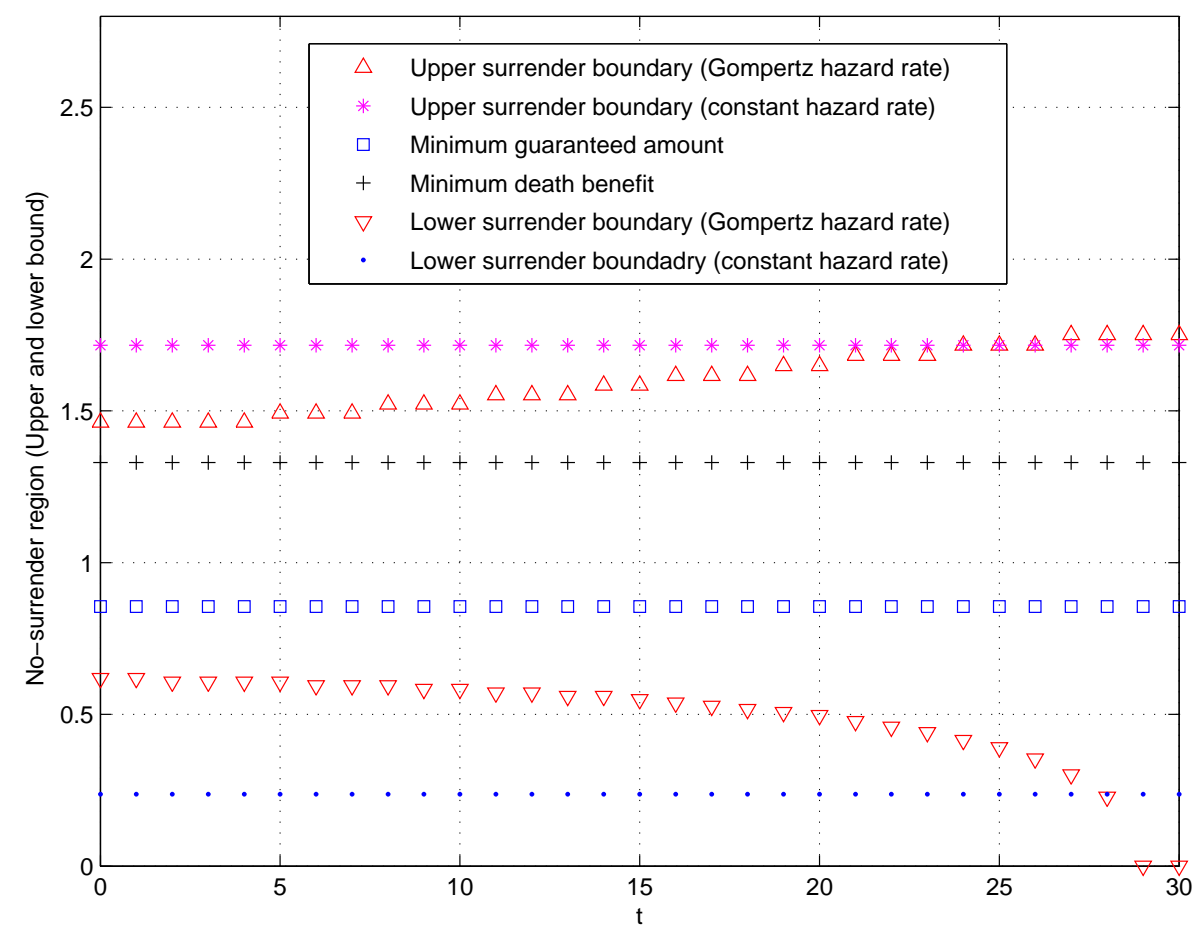

Figure 12: The Gompertz investor surrenders more readily when $t \leq 24$ and the lower free boundary vanishes for $t \geq 29$.

fund value goes, while the surrender charge is still high. For the Gompertz investor, the finite upper surrender boundary appears in $(7,8)$; under constant hazard rate, it appears in $(9,10)$. For the Gompertz investor, the future survival probabilities are higher for small $t$; thus the death benefit is less valuable than under constant hazard rate. In other words, some time between times 7 and 8, the surrender charge becomes tolerably low enough so that the Gompertz investor is willing to pay the surrender charge and forego the protection of the (less valuable) death benefit.

\section{Fixed Maturity Time}

So far, we have considered only "perpetual" EIA contracts; i.e., we have assumed that the investor may surrender the contract at any time, but she may also continue with the EIA (with the minimum guarantees) indefinitely. Considering the perpetual product allowed us to examine the qualitative behavior of the surrender boundaries; however, this is not a realistic product design. In reality, most EIA products have a fixed maturity date $T$. In this section, we consider such products. In fact, we can easily adapt our model to incorporate this feature. We find that, for the most part, the surrender boundaries for the fixed-maturity product are qualitatively similar to those for the perpetual products considered in the previous sections.

We consider two different product designs: one in which the investor is forced to sur- 


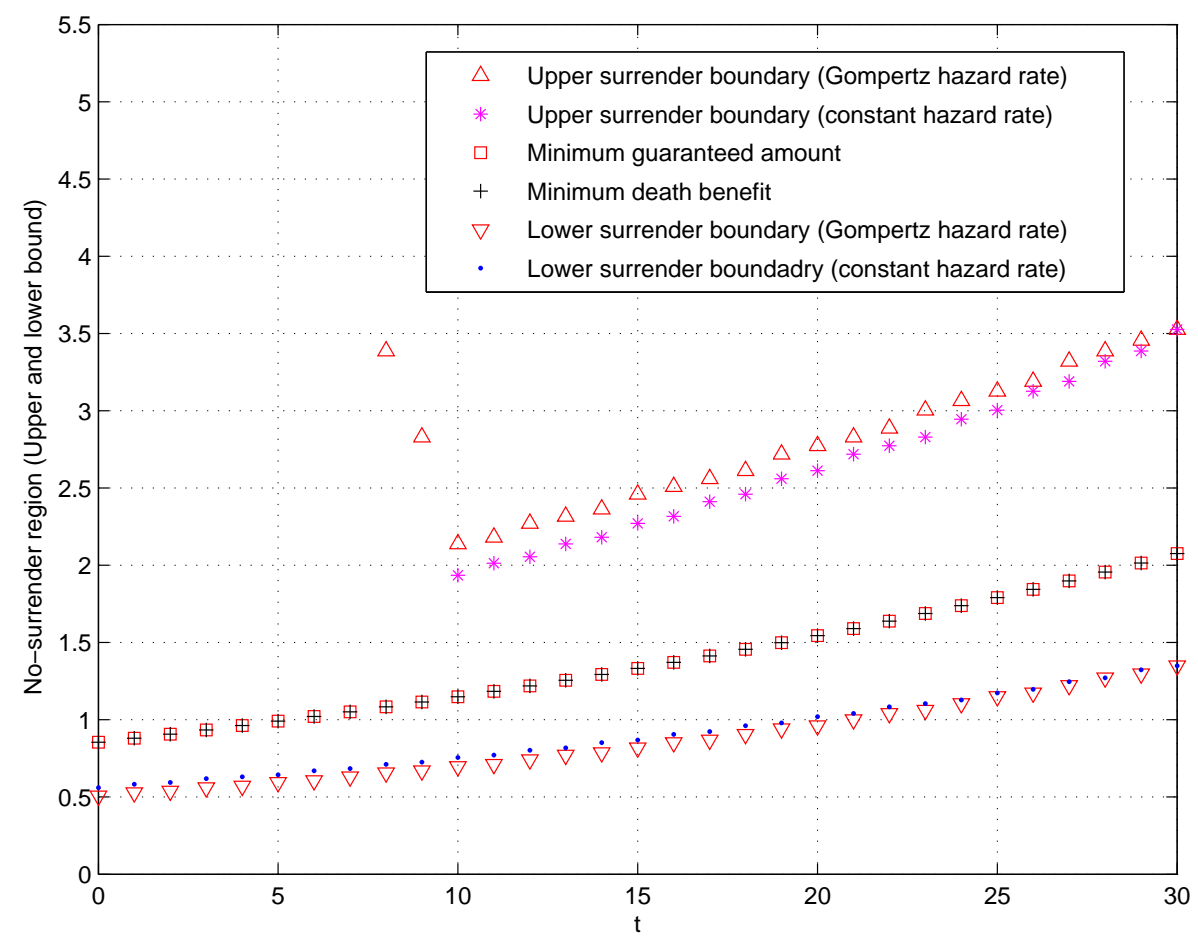

Figure 13: With time-dependent benefits, the mortality assumption has little impact on the free boundaries; the Gompertz investor is slightly more conservative.

render the contract (or roll it into a new EIA) at the maturity date $T$, and one in which the investor may continue with the EIA, but the minimum guarantee and death benefit are capped at their time $T$ levels. In other words, the guaranteed minimum growth rates are zero beyond the maturity date.

We perform one experiment for each product design. We choose the parameter values, product features, and mortality assumption as in Experiment 5.2 and contrast the surrender boundaries for the perpetual product of Experiment 5.2 with the surrender boundaries for the fixed-maturity products. The fixed maturity time coincides with the end of the surrender charge period; i.e., $T=10$.

Experiment 6.1 Forced surrender at time $T=10$ (Figure 14)

Figure 14 shows the surrender boundaries for the perpetual product (indicated by triangles) and the 10-year product (indicated by points and asterisks). We see that the surrender boundaries are close, except near the maturity date $T=10$. For the fixed maturity product, near the maturity time $T=10$, there is less uncertainty about the fund value and mortality over the remaining horizon, so the minimum guarantee and death benefit are less valuable than for the perpetual product. Thus, the investor surrenders the fixed-maturity contract more readily near the maturity date.

Experiment 6.2 Guarantees capped at time $T=10$ (Figure 15) In this experiment, we assume that the investor has the option of continuing with the EIA indefinitely, but that the guarantees are capped at their time $T$ levels. Thus, we define the 


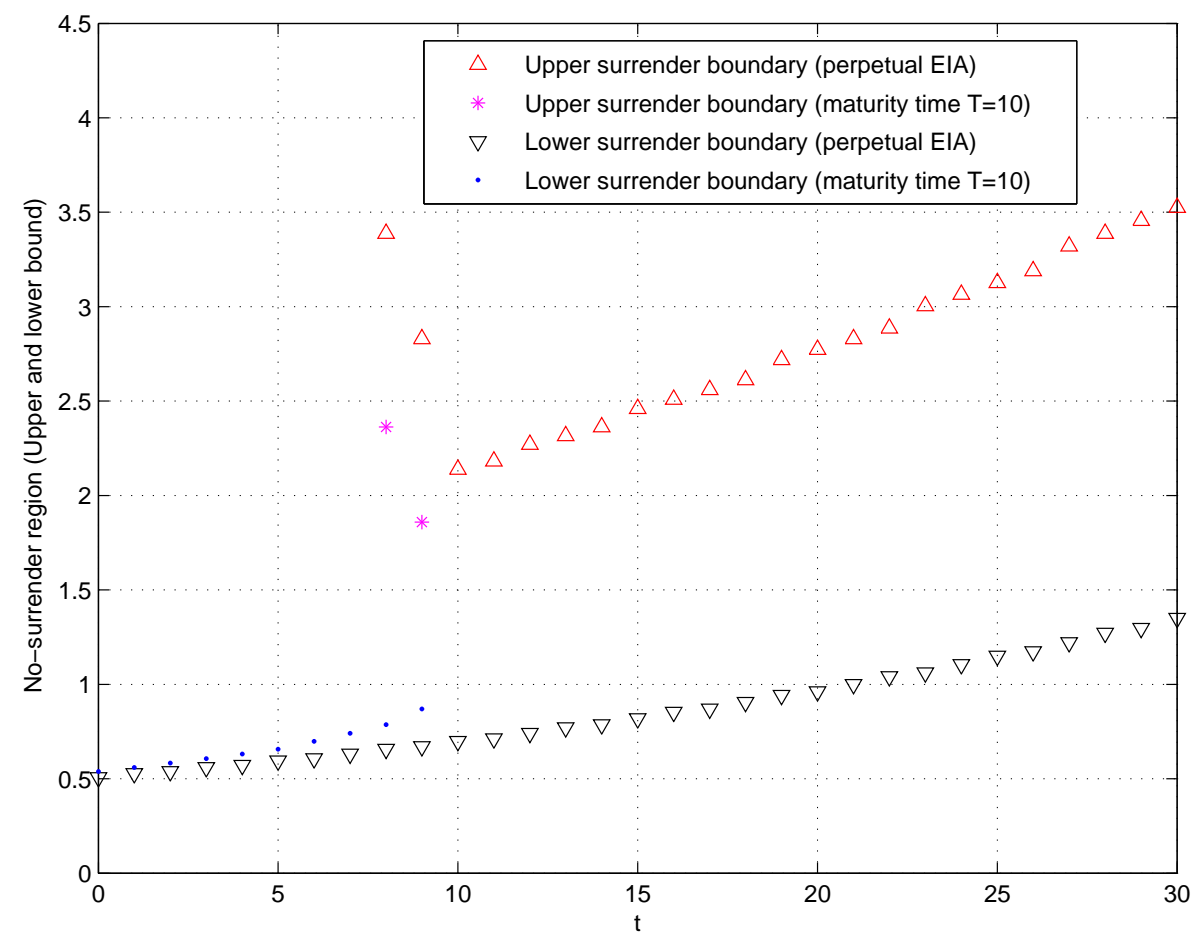

Figure 14: Near the maturity time $T=10$, there is less uncertainty about mortality and the fund value over the remaining life of the product, so the guarantees for the fixed maturity product are less valuable. Thus, the investor surrenders the fixed maturity product more readily. 


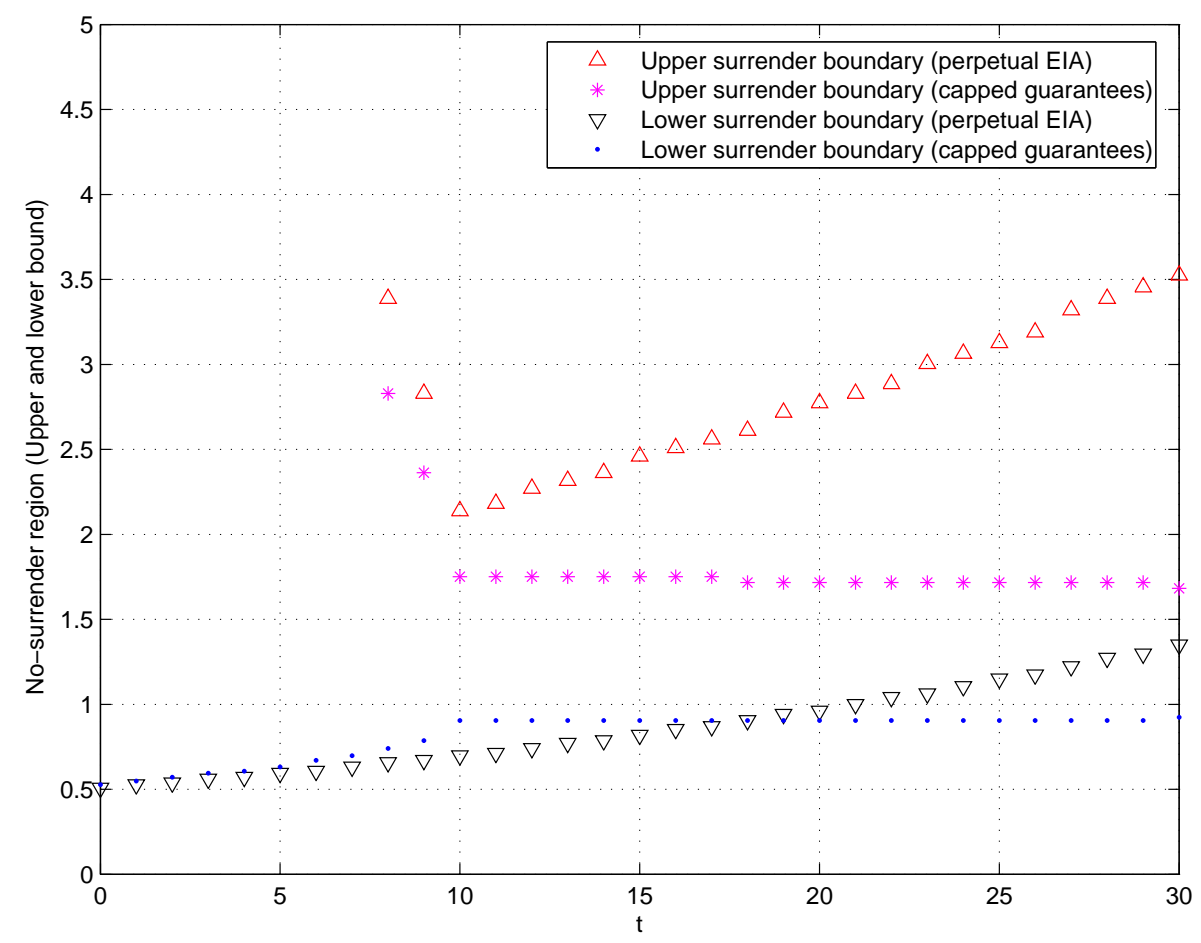

Figure 15: The surrender boundaries are constant beyond time $t=10$ because the guaranteed minimum amounts are capped at their time ten levels.

minimum guaranteed amount and death benefit by

$$
\begin{aligned}
& B\left(W_{\tau_{s}}, \tau_{s}\right)=\max \left\{0.9(1.03)^{\tau_{s}^{*}} w_{0},\left(1-f_{s}\left(\tau_{s}\right)\right) W_{\tau_{s}}\right\} \\
& D\left(W_{\tau_{d}}, \tau_{d}\right)=\max \left\{0.9(1.03)^{\tau_{d}^{*}} w_{0}, W_{\tau_{d}}\right\}
\end{aligned}
$$

where

$$
\tau_{s}^{*}=\left\{\begin{array}{cc}
\tau_{s} & \tau_{s} \leq 10 \\
10 & \tau_{s} \geq 10
\end{array}\right.
$$

and $\tau_{d}^{*}$ is defined similarly. The surrender charge $f_{s}$ is defined as in Experiment 5.2.

Figure 15 shows the surrender boundaries for the perpetual product of Experiment 5.2 (indicated by triangles) and those for the perpetual product with capped guarantees (indicated by points and asterisks). The surrender strategies are qualitatively similar but, as in Experiment 6.1, the investor with the capped guarantees surrenders more readily near the maturity time $T=10$. Moreover, for the product with capped guarantees, the surrender boundaries are constant beyond time $T=10$; this is because the minimum guaranteed amount and death benefit are constant beyond this point. For the perpetual product, the surrender boundaries continue to increase with the minimum guaranteed amount. These results are consistent with our intuition and our prior results. 


\section{Conclusion}

In this paper, we considered the optimal time for an investor to surrender an EIA contract. We examined the impact of product features, model parameters, and mortality assumptions on the optimal strategies and observed that, in some cases, there is no upper or lower surrender threshold. In the special case of time-homogeneous product features and constant hazard rate, we proved a succinct and intuitive condition on the model parameters that determines whether one or two free boundaries exist.

Several interesting directions for future work on this problem remain open; we give a partial list below.

- Is it possible to prove a characterization of the free boundary similar to Theorem 3.3 in the time-dependent case? Friedman and Shen (2002) prove this type of theorem for the optimal exercise boundary of an early retirement option.

- In Moore and Young (2005), we also considered the problem from the insurer's perspective in the time-homogenous case. Is it possible to extend this analysis to the time-dependent case?

- One might argue that utility of bequest is not the most natural objective. One might consider other optimization criteria, such as expected discounted wealth (or utility of wealth) at some fixed time $T$, such as retirement, or the probability of lifetime ruin; see, for example Milevsky, Moore, and Young (2005).

- In our analysis, we have ignored the fact that, after surrendering the contract, the investor earns the growth in the index and receives dividends. How would the inclusion of post-surrender dividends affect the optimal strategies?

- Could this analysis be extended to other indexing methods, such as the more popular annual reset method?

Acknowledgments: I gratefully acknowledge the Society of Actuaries for funding this project through a CKER Individual Grant. I especially thank Virginia Young, Fred Terry, and the participants of the 2006 Actuarial Research Day at University of Western Ontario for many fruitful discussions of this work. 


\section{Appendix 1: The Proof of Theorem 3.3}

In this section, we prove Theorem 3.3. To do so, we briefly summarize the results and notation of Section 2.2 of Moore and Young (2005) and we repeat some of the definitions and results of Section 3 for the reader's convenience.

In the no-surrender region, we wish to solve the free boundary problem (16), which we restate here:

$$
\left\{\begin{array}{c}
\left(\bar{p} \mu-f_{a}\right) w U^{\prime}+\frac{1}{2} \bar{p}^{2} \sigma^{2} w^{2} U^{\prime \prime}+\lambda u(D(w))=U(\rho+\lambda) \\
U(w) \geq V(B(w)) .
\end{array}\right\}
$$

The functions $B$ and $D$ are given by

$$
\begin{aligned}
& B(w)=\max \left\{\bar{s} w_{0}, w\right\} \quad \text { and } \\
& D(w)=\max \left\{\bar{d} w_{0}, w\right\} .
\end{aligned}
$$

It is straightforward to verify that the solution to the ODE in (26) is given by

$$
U(w)= \begin{cases}C_{1} w^{a_{1}}+C_{2} w^{a_{2}}+\frac{\lambda}{\rho+\lambda} \frac{\left(\bar{d} w_{0}\right)^{1-\gamma}}{1-\gamma}, & w \in\left(0, \bar{d} w_{0}\right) \\ \tilde{C}_{1} w^{a_{1}}+\tilde{C}_{2} w^{a_{2}}+\tilde{A} \frac{w^{1-\gamma}}{1-\gamma}, & w \geq \bar{d} w_{0}\end{cases}
$$

where $a_{1}$ and $a_{2}$ are the positive and negative roots, respectively, of

$$
\frac{1}{2} \bar{p}^{2} \sigma^{2} a^{2}+\left(\bar{p} \mu-f_{a}-\frac{1}{2} \bar{p}^{2} \sigma^{2}\right) a-(\rho+\lambda)=0
$$

and $\tilde{A}$ is given by

$$
\tilde{A}=\frac{\lambda}{\rho+\lambda-\left(\bar{p} \mu-f_{a}\right)(1-\gamma)+\bar{p}^{2} \sigma^{2} \gamma(1-\gamma) / 2} .
$$

In Moore and Young (2005), we postulated the existence of lower and upper surrender thresholds $w_{l}$ and $w_{u}$. In that paper, we computed the six unknowns $w_{l}, w_{u}, C_{i}, \tilde{C}_{i},(i=1,2)$ by assuming:

- that $U$ is continuous and smooth at $w=\bar{d} w_{0}$

- value-matching and smooth-pasting conditions at $w_{l}$ and $w_{u}$; i.e., that the value function $U$ meets the constraint function $V \circ B$ smoothly at $w_{l}$ and $w_{u}$.

These conditions gave six equations for the six unknowns. In that paper, we gave an algorithm for solving the system. The key step in the algorithm was finding a root $x=\frac{w_{u}}{w_{0}}$ of the following function:

$$
\eta(x)=\underbrace{B_{11}\left\{B_{12} x^{1-\gamma}-B_{13} x^{a_{1}}\right\}}_{\eta_{1}(x)}-\underbrace{\left[B_{21}\left\{B_{22} x^{1-\gamma}-B_{23} x^{a_{2}}\right\}^{\frac{a_{1}}{a_{2}}}\right.}_{\eta_{2}(x)} ;
$$


see equations (2.31) and (2.32) of Moore and Young (2005). From the value of $x=\frac{w_{u}}{w_{0}}$, we were able to successfully solve for $\tilde{C}_{j}, w_{l}$, and $C_{j}, j=1,2$; see Section 2.2 of Moore and Young (2005).

In this section, we prove Theorem 3.3, namely, that the existence (or non-existence) of a real root of $\eta$, and hence of two surrender boundaries, depends on the sign of $Q$, where

$$
Q=A \bar{s}^{1-\gamma}-\frac{\lambda}{\rho+\lambda} \bar{d}^{1-\gamma}
$$

and $A$ is given by

$$
A=\frac{\lambda}{\rho+\lambda-(r+m / \gamma)(1-\gamma)}
$$

We define the constants $B_{i j}$ and state our assumptions below.

Definition 8.1 We define the following:

$$
\begin{aligned}
B_{11} & =\frac{1}{a_{2} Q} \\
B_{21} & =\frac{1}{a_{1} Q} \\
B_{12} & =(A-\tilde{A})\left(a_{2}-(1-\gamma)\right) \\
B_{22} & =(A-\tilde{A})\left(a_{1}-(1-\gamma)\right) \\
B_{13} & =\left[a_{2}\left(\frac{\lambda}{\rho+\lambda}-\tilde{A}\right)+\tilde{A}(1-\gamma)\right] \bar{d}^{1-\gamma-a_{1}} \\
B_{23} & =\left[a_{1}\left(\frac{\lambda}{\rho+\lambda}-\tilde{A}\right)+\tilde{A}(1-\gamma)\right] \bar{d}^{1-\gamma-a_{2}} .
\end{aligned}
$$

Assumption 8.2 To prove each of the three parts of Theorem 3.3, we must assume some of the conditions below.

(A1): $B_{12}>0$

(A2): $B_{13}, B_{22}, B_{23}<0$

(A3): $a_{2}<1-\gamma$

(A4): $B_{23}<B_{22}<0$

$(A 5): a_{1}>1$

Remark 8.3 Assumptions (A1)-(A4) are innocuous for "realistic" values of the model parameters; they held for every "realistic" choice of parameters that we tested. On the other hand, the order of $B_{13}$ and $B_{23}$ varies for "realistic" examples; thus, we do not impose an assumption on the ordering of these constants.

Since $a_{1}$ is the positive root of the quadratic function given in (28), assuming that $a_{1}>1$ is equivalent to assuming that $\bar{p} \mu-f_{a}-(\rho+\lambda)<0$; i.e., that the drift on the index (adjusted for the participation rate and fees) is smaller than the investor's mortality-adjusted discount rate. This assumption also held for all of our "realistic" choices of model parameters. 
We prove Theorem 3.3 in three parts, as Theorems 8.4 through 8.6 below.

Theorem 8.4 Assume that $\gamma>1$ and that the model parameters satisfy the conditions (A1) and (A2) of Assumption 8.2. Suppose $Q>0$. Then there is no real root of the function $\eta$ given in (29).

Proof: $\quad$ If $Q>0$, then $B_{11}<0$ and $B_{21}>0$. Assumptions (A1) and (A2) imply that $\eta_{1}<0$ on $(0, \infty)$. Since $a_{1} / a_{2}<0, \eta_{2}$ is either positive or non-real. In either case, since $\eta_{1}<0, \eta$ has no real root.

Theorem 8.5 Assume that $\gamma>1$ and that the model parameters satisfy the conditions (A1)-(A4) in Assumption 8.2. Suppose $Q<0$. Then the function $\eta$ given in (29) has a real root $x>1$.

Proof: $\quad$ Note that $\eta$ has a root if and only if there exists an $x$ such that $\eta_{1}(x)=\eta_{2}(x)$, where $\eta_{1}$ and $\eta_{2}$ are defined in (29). The idea of the proof is quite simple:

- we show that there exists an $x^{*}>1$ such that $\eta_{2}\left(x^{*}\right)=\infty$

- we show that there exists an $\tilde{x}>x^{*}$ such that $\eta_{1}(\tilde{x})>\eta_{2}(\tilde{x})$ and

- we use the continuity of $\eta_{1}$ and $\eta_{2}$ on $\left(x^{*}, \tilde{x}\right)$ to conclude that the graphs of $\eta_{1}$ and $\eta_{2}$ must cross in $\left(x^{*}, \tilde{x}\right)$.

That is the simple idea of the proof; the details follow. Figure 16 shows the qualitative properties of the functions $\eta_{1}, \eta_{2}, \eta_{1}^{a}$, and $\eta_{2}^{a}$; the reader might find it helpful to refer to this figure occasionally while reading through the proof.

Note first that since $Q<0$, we have that $B_{11}>0$ and $B_{21}<0$. Define

$$
\tilde{\eta}_{2}(x)=\eta_{2}(x)^{\frac{a_{2}}{a_{1}}}=B_{21}\left\{B_{22} x^{1-\gamma}-B_{23} x^{a_{2}}\right\}
$$

and note that $\tilde{\eta}_{2}$ has a zero at

$$
x=x^{*}:=\left(\frac{B_{23}}{B_{22}}\right)^{\frac{1}{1-\gamma-a 2}} ;
$$

by Assumptions (A2)-(A4), $x^{*}>1$.

Assumption (A4) implies that $\tilde{\eta}_{2}(1)<0$; thus $\tilde{\eta}_{2}<0$ on $\left(0, x^{*}\right)$. It follows that $\eta_{2} \notin \mathbf{R}$ on $\left(0, x^{*}\right)$. We claim that $\tilde{\eta}_{2}>0$ on $\left(x^{*}, \infty\right)$. To prove this, we find a "special" $x_{c}>x^{*}$ such that $\tilde{\eta}\left(x_{c}\right)>0$. Since $\tilde{\eta}$ is continuous and has no other roots on $\left(x^{*}, \infty\right)$, if follows that $\eta_{2}>0$ on $\left(x^{*}, \infty\right)$.

Let $x_{c}$ be the critical point of $\tilde{\eta}_{2}$; i.e.,

$$
x_{c}=\left[\frac{B_{22}(1-\gamma)}{B_{23} a_{2}}\right]^{\frac{1}{\gamma-1+a 2}} .
$$


Note that

$$
x_{c}=\left(\frac{B_{22}}{B_{23}}\right)^{\frac{1}{\gamma-1+a 2}}\left(\frac{1-\gamma}{a_{2}}\right)^{\frac{1}{\gamma-1+a 2}}=\left(\frac{B_{22}}{B_{23}}\right)^{\frac{1}{\gamma-1+a 2}} \ell=x^{*} \ell .
$$

Also, by Assumption (A3), $\ell>1$; therefore, $x^{*}<x_{c}$.

We claim that $\tilde{\eta}_{2}\left(x_{c}\right)>0$. To see this, note that

$$
\begin{aligned}
\frac{\tilde{\eta}_{2}\left(x_{c}\right)}{B_{21}} & =B_{22} x_{c}^{1-\gamma}-B_{23} x_{c}^{a_{2}} \\
& =B_{22}\left(x^{*} \ell\right)^{1-\gamma}-B_{23}\left(x^{*} \ell\right)^{a_{2}} \\
& =\ell^{1-\gamma}\left[B_{22} x^{* 1-\gamma}-B_{23} x^{* a_{2}} \ell^{a_{2}-1+\gamma}\right] .
\end{aligned}
$$

Recall that $x^{*}$ is a zero of $\tilde{\eta}_{2}$; thus, by Assumptions (A2) and (A3), we have that

$$
B_{22} x^{* 1-\gamma}=B_{23} x^{* a_{2}}<B_{23} x^{* a_{2}} \ell^{a_{2}-1+\gamma},
$$

since $\ell^{a_{2}-1+\gamma}<1$. Therefore, $\frac{\tilde{\eta}_{2}\left(x_{c}\right)}{B_{21}}<0$ and, since $Q<0$ implies that $B_{21}<0$, we have that $\tilde{\eta}_{2}\left(x_{c}\right)>0$.

Thus, on $\left(x^{*}, x_{c}\right)$, we have the following:

- $\tilde{\eta}_{2}>0$ and therefore $\eta_{2}>0$

- $\tilde{\eta}_{2} \rightarrow 0$ as $x \rightarrow x^{*}$ and as $x \rightarrow \infty$

- $\eta_{2} \rightarrow \infty$ as $x \rightarrow x^{*}$ and as $x \rightarrow \infty$

- $x=x_{c}$ is a global minimum of $\eta_{2}$;

see the middle graph in Figure 16.

To show that $\eta$ has a zero on $\left(x^{*}, \infty\right)$, we first note that $Q<0,(\mathrm{~A} 1)$, and (A3) together imply that $\eta_{1} \geq 0$ on $(0, \infty)$. Since $\eta_{2} \rightarrow \infty$ at $x=x^{*}$, we have that $\eta_{1}<\eta_{2}$ near $x=x^{*}$. We claim that there exists an $\tilde{x}>x^{*}$ such that $\eta_{1}(\tilde{x})>\eta_{2}(\tilde{x})$; thus, the graphs of $\eta_{1}$ and $\eta_{2}$ cross in $\left(x^{*}, \tilde{x}\right)$. The crossing point is a zero of $\eta$.

Note that for large $x$, by (A2)-(A4) we have

$$
\begin{aligned}
& \eta_{1}(x) \approx \eta_{1}^{a}(x):=-B_{11} B_{13} x^{a_{1}}<\eta_{1}(x) \\
& \eta_{2}(x) \approx \eta_{2}^{a}(x):=\left[B_{21} B_{22} x^{1-\gamma}\right]^{\frac{a 1}{a 2}}<\eta_{2}(x)
\end{aligned}
$$

$\eta_{j}^{a}$ are the dominant terms (or asymptotic limits) of $\eta_{j}, j=1,2$; see the bottom graph in Figure 16. We claim that $\eta_{1}^{a}>\eta_{2}^{a}$ for large $x$. This is simply because $\eta_{1}^{a}$ is a higher power of $x$. To make this more precise, observe that for large $x$, (A3) implies that

$$
\underbrace{\left(-B_{11} B_{13}\right)^{\frac{1}{a_{1}}} x}_{\eta_{1}^{a}(x)^{\frac{1}{a_{1}}}}>\underbrace{\left(B_{21} B_{22}\right)^{\frac{1}{a_{2}}} x^{\frac{1-\gamma}{a_{2}}}}_{\eta_{2}^{a}(x)^{\frac{1}{a_{1}}}} .
$$

It follows then that $\eta_{1}^{a}(x)>\eta_{2}^{a}(x)$ for large $x$. Moreover, the distance between $\eta_{1}^{a}$ and $\eta_{2}^{a}$ increases with $x$; see the third graph in Figure 16. 

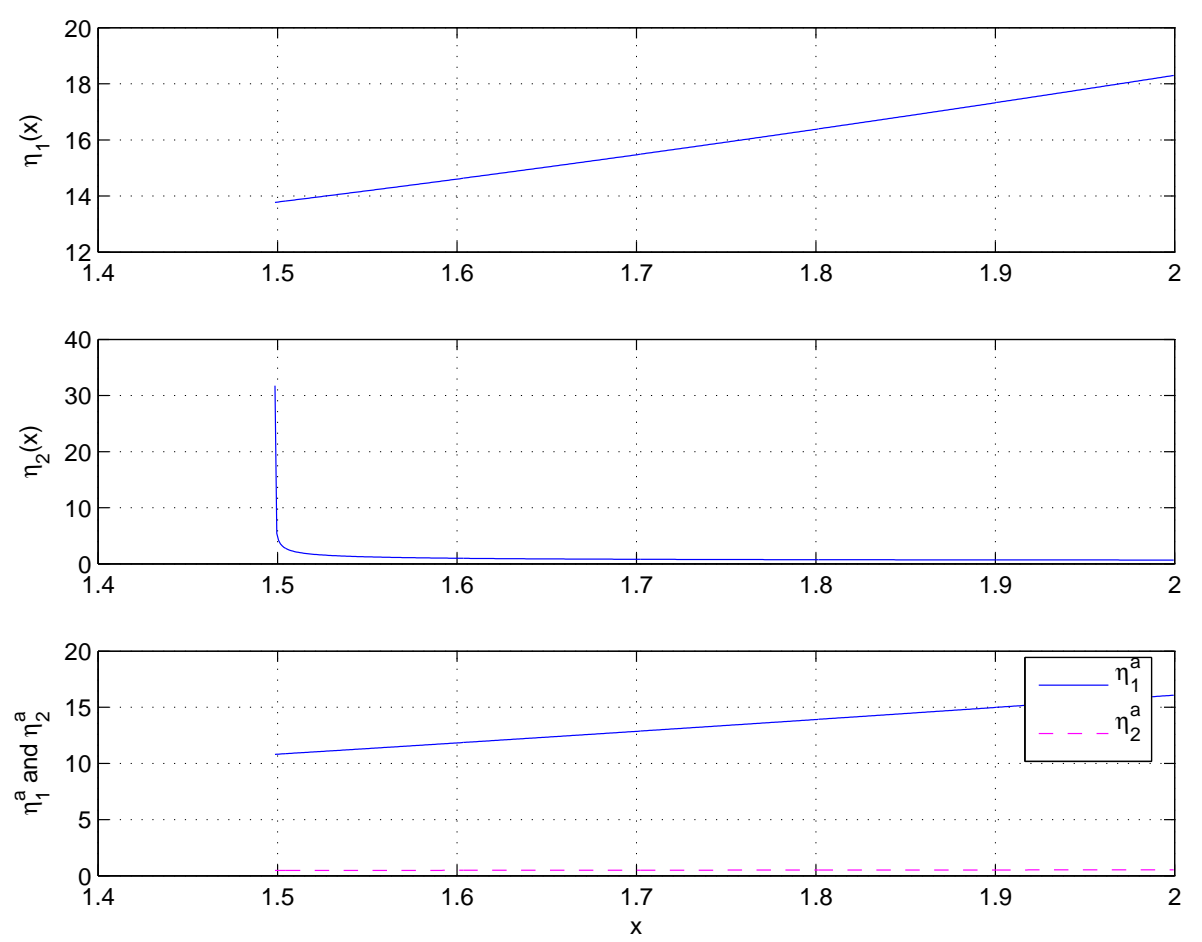

Figure 16: In the middle graph, we see that $\eta_{2}$ has a vertical asymptote at $x=x^{*} \approx 1.50$. The top graph shows that $\eta_{1}$ is eventually greater than $\eta_{2}$. We can make this argument precise by considering $\eta_{1}^{a}$ and $\eta_{2}^{a}$ for "large" $x$.

We have shown that the graph of $\eta_{1}$ starts out below the graph of $\eta_{2}$ and the (approximate) graph of $\eta_{1}$ is eventually above the (approximate) graph of $\eta_{2}$. We conclude that $\eta_{1}$ is eventually greater than $\eta_{2}$. To make this a precise argument, choose $\varepsilon>0$. Since $\eta_{1}-\eta_{1}^{a} \rightarrow 0, \eta_{2}-\eta_{2}^{a} \rightarrow 0$, and the distance between $\eta_{1}^{a}$ and $\eta_{2}^{a}$ increases with $x$, we can choose $x_{1}, x_{2}$, and $x_{3}$ to satisfy the following:

- $\eta_{1}-\eta_{1}^{a}<\varepsilon$ for $x>x_{1}$;

- $\eta_{2}-\eta_{2}^{a}<\varepsilon$ for $x>x_{2}$;

- $\eta_{1}^{a}-\eta_{2}^{a}>3 \varepsilon$ for $x>x_{3}$.

Let $\tilde{x}=\max \left\{x_{1}, x_{2}, x_{3}\right\}$. Then at $x=\tilde{x}$, we have

$$
\begin{aligned}
\eta_{1}-\eta_{2} & =\eta_{1}-\eta_{1}^{a}+\eta_{1}^{a}-\left(\eta_{2}-\eta_{2}^{a}+\eta_{2}^{a}\right) \\
& =\underbrace{\eta_{1}-\eta_{1}^{a}}_{\in(0, \varepsilon)}-\underbrace{\left(\eta_{2}-\eta_{2}^{a}\right)}_{\in(0, \varepsilon)}+\underbrace{\eta_{1}^{a}-\eta_{2}^{a}}_{>3 \varepsilon} \\
& \geq 0-\varepsilon+3 \varepsilon=2 \varepsilon .
\end{aligned}
$$

Since $\eta_{1}>\eta_{2}$ at $x=\tilde{x}$, there must be a zero of $\eta$ in $\left(x^{*}, \tilde{x}\right)$; the proof is complete. 
Theorem 8.6 Assume that $\gamma>1$ and that condition (A5) of Assumption 8.2 holds. Suppose $Q=0$. Then the lower surrender threshold occurs at $w_{l}=0$.

Proof: $\quad$ Set $C_{2}=0$ in the solution (27) to the $\operatorname{ODE}(26)$, so that for $w \in\left(0, \bar{d} w_{0}\right)$,

$$
U(w)=C_{1} w^{a_{1}}+\frac{\lambda}{\rho+\lambda} \frac{\left(\bar{d} w_{0}\right)^{1-\gamma}}{1-\gamma} .
$$

Then it is easy to verify that when $Q=0$,

$$
U(0)=\frac{\lambda}{\rho+\lambda} \frac{\left(\bar{d} w_{0}\right)^{1-\gamma}}{1-\gamma}=A \frac{\left(\bar{s} w_{0}\right)^{1-\gamma}}{1-\gamma}=(V \circ B)(0)
$$

and that

$$
\left.\frac{d}{d w} U(w)\right|_{w=0}=\left.C_{1} w^{a_{1}-1}\right|_{w=0}=0=\frac{d}{d w}(V \circ B)(0) ;
$$

thus, the value function $\mathrm{U}$ meets the constraint function $V \circ B$ smoothly at $w=0$. Note that we used the fact that $a_{1}>1$ in computing the $U^{\prime}(0)$ and that we did not need any of the other conditions of Assumption 8.2. 


\section{References}

[1] 2005 Fourth Quarter Index Annuity Sales (3/06). The Advantage Compendium. http://www.indexannuity.org/library.htm (Sales).

[2] Campell, J. Y. and L. M. Viceira (2002), Strategic Asset Allocation, Oxford University Press, Oxford.

[3] Cheung, K. C. and H. Yang (2005), Optimal stopping behavior of equity-linked investment products with regime switching, Insurance: Mathematics and Economics, 37: 599-614.

[4] Cochrane, J. (2001), Asset Pricing, Princeton University Press, Princeton, New Jersey.

[5] Feldstein, M. and E. Ranguelova (2001), Individual risk in an investment-based social security system, American Economic Review, 91 (4): 1116-1125.

[6] Friedman, A. and W. Shen (2002), A variational inequality approach to financial valuation of retirement benefits based on salary, Finance and Stochastics, 6 (3): 273302.

[7] Friend, I. and M. E. Blume (1975), The demand for risky assets, American Economic Review, 65: 900-922.

[8] Fung, H. and L. K. Li (2003), Pricing discrete dynamic fund protections, North American Actuarial Journal, 7 (4): 23-31.

[9] Gerber, H. U. and G. Pafumi (2000), Pricing dynamic investment fund protection, North American Actuarial Journal, 4 (2): 28-37; Discussions 37-41 and 5 (1) (2001): 153-157.

[10] Gerber, H. U. and E. S. W. Shiu (2003), Pricing perpetual fund protection with withdrawal option, North American Actuarial Journal, 7 (2): 60-77; Discussions 77-92 and 8 (1) (2004): 96-99.

[11] Hardy, M. (2003), Investment Guarantees: The New Science of Modeling and Risk Management for Equity-Linked Insurance, John Wiley, New York.

[12] Huang, H., M. A. Milevsky, and J. Wang (2004), Ruined moments in your life: how good are the approximations?, Insurance: Mathematics and Economics, 34 (3): 421447.

[13] Imai, J. and P. P. Boyle (2001), Dynamic fund protection, North American Actuarial Journal, 5 (3): 31-49; Discussion 49-51.

[14] Jeanblanc, M., P. Lakner, and A. Kadam (2004), Optimal bankruptcy time and consumption/investment policies on an infinite horizon with a continuous debt repayment until bankruptcy, Mathematics of Operations Research, 29 (3): 649-671. 
[15] Krylov, N. V. (1980), Controlled Diffusion Processes, Springer-Verlag, Berlin.

[16] Lee, H. (2002), Pricing exotic options with applications to equity-indexed annuities. Ph. D. thesis, University of Iowa, Iowa City.

[17] Lin, X. S. and K. S. Tan (2003), Valuation of equity-indexed annuities under stochastic interest rates, North American Actuarial Journal, 7 (4): 72-91.

[18] Merton, R. C. (1992), Continuous-Time Finance, revised edition, Blackwell Publishers, Cambridge, Massachusetts.

[19] Milevsky, M. A., K. S. Moore, and V. R. Young (2005), Asset allocation and annuitypurchase strategies to minimize the probability of financial ruin. Mathematical Finance, to appear.

[20] Milevsky, M. A. and V. R. Young. Annuitization and asset allocation (2003), Working paper, Individual Finance and Insurance Decisions Centre, Schulich School of Business, York University.

[21] Mitchell, O. S., J. M. Poterba, M. J. Warshawsky, and J. R. Brown (1999), New evidence on the moneys worth of individual annuities, American Economic Review, December, 89 (5): 1299-1318.

[22] Moore, K. S. and V. R. Young (2005), Optimal design of a perpetual equity-indexed annuity, North American Actuarial Journal, 9 (1): 57-72.

[23] Moore, K. S. and V. R. Young (2003), Pricing equity-indexed pure endowments via the principle of equivalent utility, Insurance: Mathematics and Economics, 33: 497-516.

[24] Øksendal, B. (2000), Stochastic Differential Equations: An Introduction with Applications, fifth edition, Springer-Verlag, Berlin.

[25] Pratt, J. W. (1964), Risk aversion in the small and in the large, Econometrica, 32: 122-136.

[26] Tiong, S. (2000), Valuing equity-indexed annuities, North American Actuarial Journal, 4 (4): 149-163; Discussions 163-170 and 5 (3) (2001): 133-136.

[27] Wilmott, P., J. Dewynne, and S. Howison (2000), Option Pricing: Mathematical Models and Computation, Oxford Financial Press, Oxford.

[28] Young, V. R. (2003), Equity-indexed life insurance: Pricing and reserving using the principle of equivalent utility, North American Actuarial Journal, 7 (1): 68-86.

[29] Young, V. R. and T. Zariphopoulou (2002), Pricing dynamic insurance risks using the principle of equivalent utility, Scandinavian Actuarial Journal, (4): 246-279. 